Corrected: Publisher correction

ARTICLE

https://doi.org/10.1038/s41467-019-12056-1

\title{
Carrier lifetime enhancement in halide perovskite via remote epitaxy
}

Jie Jiang (1) 1,2,9, Xin Sun (10) 3,9, Xinchun Chen (1) ${ }^{4}$, Baiwei Wang (1) 2, Zhizhong Chen², Yang Hu ${ }^{2}$, Yuwei Guo²,

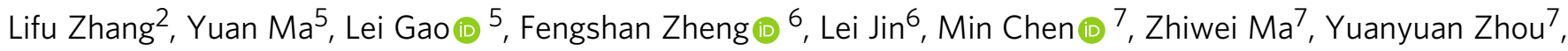
Nitin P. Padture (i) ${ }^{7}$, Kory Beach (10) ${ }^{3}$, Humberto Terrones (10 ${ }^{3}$, Yunfeng Shi ${ }^{2}$, Daniel Gall ${ }^{2}{ }^{2}$, Toh-Ming Lu ${ }^{3}$, Esther Wertz (i) ${ }^{3}$, Jing Feng ${ }^{1} \&$ Jian Shi (i) ${ }^{2,8}$

Crystallographic dislocation has been well-known to be one of the major causes responsible for the unfavorable carrier dynamics in conventional semiconductor devices. Halide perovskite has exhibited promising applications in optoelectronic devices. However, how dislocation impacts its carrier dynamics in the 'defects-tolerant' halide perovskite is largely unknown. Here, via a remote epitaxy approach using polar substrates coated with graphene, we synthesize epitaxial halide perovskite with controlled dislocation density. First-principle calculations and molecular-dynamics simulations reveal weak film-substrate interaction and low density dislocation mechanism in remote epitaxy, respectively. High-resolution transmission electron microscopy, high-resolution atomic force microscopy and Cs-corrected scanning transmission electron microscopy unveil the lattice/atomic and dislocation structure of the remote epitaxial film. The controlling of dislocation density enables the unveiling of the dislocation-carrier dynamic relation in halide perovskite. The study provides an avenue to develop free-standing halide perovskite film with low dislocation density and improved carried dynamics.

\footnotetext{
${ }^{1}$ Department of Materials Science and Engineering, Kunming University of Science and Technology, Kunming, Yunnan 650093, China. ${ }^{2}$ Department of Materials Science and Engineering, Rensselaer Polytechnic Institute, Troy 12180, United States. ${ }^{3}$ Department of Physics, Applied Physics and Astronomy, Rensselaer Polytechnic Institute, Troy, NY 12180, USA. ${ }^{4}$ State Key Laboratory of Tribology, Tsinghua University, Beijing 100084, China. ${ }^{5}$ Beijing Advanced Innovation Center for Materials Genome Engineering, Institute for Advanced Materials and Technology, University of Science and Technology Beijing, Beijing 100083, China. ${ }^{6}$ Ernst Ruska-Centre for Microscopy and Spectroscopy with Electrons and Peter Grünberg Institute, Forschungszentrum Jülich, Jülich, Germany. ${ }^{7}$ School of Engineering, Brown University, Providence, RI 02912, USA. ${ }^{8}$ Center for Materials, Devices, and Integrated Systems, Rensselaer Polytechnic Institute, Troy, NY 12180, United States. ${ }^{9}$ These authors contributed equally: Jie Jiang, Xin Sun. Correspondence and requests for materials should be addressed to L.G. (email: gaolei@ustb.edu.cn) or to J.F. (email: vdmzsfj@qq.com) or to J.S. (email: shij4@rpi.edu)
} 
$\mathrm{D}$ islocation, a crystallographic defect in a crystal structure, is well-known to strongly affect the material's physical properties. Electron-hole recombination mediated via dislocations (e.g., threading dislocation) is one of the predominant loss mechanisms for the sub-optimum performance in semiconductors devices. Typical examples include the quantum efficiency loss in GaN-based light-emitting diodes (LEDs) ${ }^{1,2}$ and GaAs solar cells ${ }^{3}$. The relationship between dislocation and carrier dynamic has been well experimentally investigated and understood in conventional semiconductors such as $\mathrm{GaN}^{4}$ and $\mathrm{Si}^{5}$. Recently, halide perovskite has been showing great promises 6,7 in many semiconductor devices such as photovoltaics $^{8-10}$, LEDs ${ }^{11,12}$, lasers ${ }^{13-15}$, photodetectors ${ }^{16,17}$ and transistors 18,19 . Despite their 'defects-tolerant' nature ${ }^{20-22}$, the effects of grain boundary ${ }^{23-27}$, interfaces ${ }^{16,28,29}$, points defects $^{8,30-32}$, and phase impurity ${ }^{33-35}$ in halide perovskite on the carrier dynamics and device performances have been widely recognized and studied ${ }^{31,36-39}$. However, its dislocation-carrier dynamic relation has never been unveiled. Whether dislocation could severely disturb halide perovskite's carrier dynamics has been remaining a puzzle. The lack of dislocation-carrier dynamic study has been mainly due to the inability in controlling dislocation configurations and quantifies.

Recently, monolayer graphene was introduced as a buffer layer for epitaxial growth of semiconductor materials such as $\mathrm{GaAs}^{40,41}$. With this concept, remote heteroepitaxy was achieved in the systems of AlN film on sapphire ${ }^{42}$, copper film on sapphire ${ }^{43}$, and $\mathrm{ZnO}$ film on $\mathrm{GaN}^{44}$. In these studies, it is believed that the graphene buffer layer screens the majority of the substrate electrostatic potential but still allows a weak substrate-film coupling ${ }^{45,46}$. In addition to graphene transparency, the strong polarity of substrates that provides long-distance electrostatic decaying potential is believed to be the other substantial factor enabling remote epitaxy ${ }^{40,41}$. In this report, by taking advantages of the concept of remote epitaxy and its ability in controlling filmsubstrate coupling and further regulating dislocation densities (misfit and then threading), we have successfully epitaxially grown halide perovskite crystals and films of controlled dislocations (derived from the density of etching rosettes) on two strong polar $\mathrm{NaCl}$ and $\mathrm{CaF}_{2}$ substrates with monolayer graphene buffered. Density functional theory (DFT) calculations have revealed the structure and magnitude of the incompletely screened electrostatic potential from the polar substrates, supporting the remote epitaxy in the present case. The regulated film-substrate interactions have further reflected themselves in controlling the wavelengths of the ferroelastic domains. Molecular-dynamics (MD) simulations reveal the kinetic process during remote epitaxy. Comparing to the ionic epitaxy with high dislocation density (both misfit and threading), the film grown via remote shows much enhanced photoluminescence intensity and increased carrier lifetime. Our successful demonstration of remote epitaxy in halide perovskite provides an approach to develop free-standing halide perovskite film with reduced dislocation density. More importantly, dislocations and their impacts on carrier dynamics and device performance in halide perovskite have to be recognized and scrutinized.

\section{Results}

Epitaxy relation and lattice structure. Before growth, graphene layers are transferred onto $\mathrm{NaCl}(001)$ and $\mathrm{CaF}_{2}(001)$ as substrates $(\mathrm{Gr} / \mathrm{NaCl}(001)$ and $\mathrm{Gr} / \mathrm{NaCl}(001))$ for remote epitaxy. Optical images in Supplementary Fig. 1a and e show the surface morphology of $\mathrm{Gr} / \mathrm{NaCl}(001)$ and $\mathrm{Gr} / \mathrm{NaCl}(001)$. Both defected region $^{47}$ (low intensity ratio of $2 \mathrm{D}$ and G bands in Supplementary Fig. $1 c$ and $g$, and high intensity of $D$ and $G$ bands in
Supplementary Fig. 1d and h) and good region (high intensity ratio of 2D and G bands in Supplementary Fig. 1c and g, and low intensity ratio of D and G bands in Supplementary Fig. $1 \mathrm{~d}$ and $\mathrm{h}$ ) of graphene on substrates are characterized by Raman spectra and mappings, as shown in Supplementary Fig. $1 \mathrm{~b}-\mathrm{d}$ and $\mathrm{f}-\mathrm{h}$. The atomic-scale structures of graphene surface on both $\mathrm{NaCl}(001)$ and $\mathrm{CaF}_{2}(001)$ substrates are investigated by high resolution atomic force microscopy (HRAFM), as shown in Supplementary Fig. $2 \mathrm{a}$ and $\mathrm{c}$, respectively. Supplementary Fig. $2 \mathrm{~b}$ and $\mathrm{d}$ show the fast Fourier transforms (FFT) and the bright spots can be indexed to graphene structure. The spots splitting in Supplementary Fig. $2 \mathrm{~b}$ and the unclear additional spots in Supplementary Fig. 2d could be induced by the chemical potential influence from the substrates underneath.

Chemical vapor deposition (CVD) method was used to achieve both ionic and remote epitaxy (more details can be found in Methods, Characterizations in Supplementary Information). Under optimized growth condition, two typical samples were grown, including $\mathrm{CsPbBr}_{3}$ thin film grown on $\mathrm{NaCl}(001)$ substrate $\left(\mathrm{CsPbBr} / \mathrm{NaCl}\right.$, i.e., ionic epitaxy) and $\mathrm{CsPbBr}_{3}$ flakes grown on monolayer graphene buffered $\mathrm{NaCl}(001)$ substrate $\left(\mathrm{CsPBBr}_{3} / \mathrm{Gr} /\right.$ $\mathrm{NaCl}$, i.e., remote epitaxy). The crystallinity and the epitaxial relationship of $\mathrm{CsPbBr}_{3}$ with substrates were characterized by $\mathrm{X}$ ray diffraction (XRD) high-resolution reciprocal space mapping (RSM). Figure 1a, b shows the RSM of both 224 peaks for as-grown $\mathrm{CsPbBr}_{3}$ and $\mathrm{NaCl}$ substrate in two typical samples, $\mathrm{CsPBBr}_{3} / \mathrm{NaCl}$ and $\mathrm{CsPbBr}_{3} / \mathrm{Gr} / \mathrm{NaCl}$. Two types of peaks of $\mathrm{CsPbBr}_{3}$ for both samples could be found (Fig. 1a, b and Supplementary Fig. 3: XRD $\omega-2 \theta$ scans for both samples). Based on the four-fold symmetry exhibited in the X-ray pole figure in Fig. 1c, the crystal structure for the as-grown samples could be assigned to tetragonal structure (P4/ $\mathrm{mbm}$ ) or orthorhombic structure (Pbnm or Pmna) due to the close lattice constants of $a(8.207 \AA)$ and $b(8.255 \AA)$ in orthorhombic $(\mathrm{Pbnm}) \mathrm{phase}^{48,49}$. It should be noted that, for convenience, in some analysis, $\mathrm{CsPbBr}$ is indexed with pseudocubic structure. The epitaxial relationships for both samples are consistent and could be interpreted as out-of-plane $\mathrm{CsPbBr}_{3}(001) \| \mathrm{NaCl}(001)$ and in-plane $\mathrm{CsPbBr}_{3}[100] \| \mathrm{NaCl}[100]$. The lattice constants of $\mathrm{CsPbBr}_{3}$ are calculated to be $a_{\|}=5.811 \AA, a_{\perp}=5.830 \AA$ and $a_{\|}=5.817 \AA$, $a_{\perp}=5.850 \AA$ for $\mathrm{CsPbBr}_{3} / \mathrm{NaCl}$ and $\mathrm{CsPbBr}_{3} / \mathrm{Gr} / \mathrm{NaCl}$, respectively. As shown in the insets of Supplementary Fig. 3, high crystallinity for both samples in terms of rocking curves of 004 peaks is obtained even on the multi-domains $\mathrm{NaCl}$ substrates. The better crystallinity and the decrease in the full width at half maximum (FWHM) of the rocking curves in the remote epitaxial sample comparing to the ionic epitaxial one (from $0.39^{\circ}$ to $0.29^{\circ}$ ) were characterized. The in-plane orientations of the remote and ionic epitaxial samples can also be revealed by X-ray pole figures of $\mathrm{CsPbBr}_{3} 111$ and $\mathrm{NaCl}$ 111, as shown in Fig. 1c and Supplementary Fig. 4, respectively.

Although the same growth conditions have been applied for both samples, the remote epitaxial one exhibits totally different morphology (flakes), as can be seen in the scanning electron microscope (SEM) image Fig. 1e, due to the remarkable change of the growth thermodynamics and kinetics for remote epitaxy. The flake size varies from less than $1 \mu \mathrm{m}$ to more than $10 \mu \mathrm{m}$, due to the successive epitaxial nucleation which is typical for the halide perovskite epitaxial growth ${ }^{50}$. The size of $\mathrm{CsPbBr}_{3}$ flake and thickness of thin film can be tuned by growth time and substrate temperature. Typical cross-sectional SEM images of the ionic epitaxial film with thickness of around $1 \mu \mathrm{m}$ and the remote epitaxial films with thickness of around $1.5 \mu \mathrm{m}, 1 \mu \mathrm{m}$ and $2.2 \mu \mathrm{m}$, are shown in Supplementary Fig. 5a, Fig. 1f, Supplementary Fig. $5 b, c$, respectively. Atomic force microscopy (AFM) images of Supplementary Fig. 6a, b show smooth surface of ionic and remote epitaxial flakes with surface root mean square (RMS) 


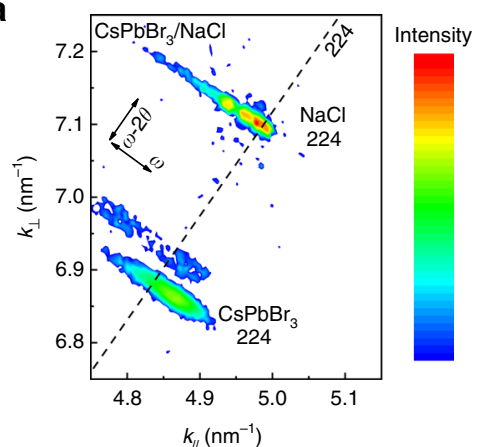

C

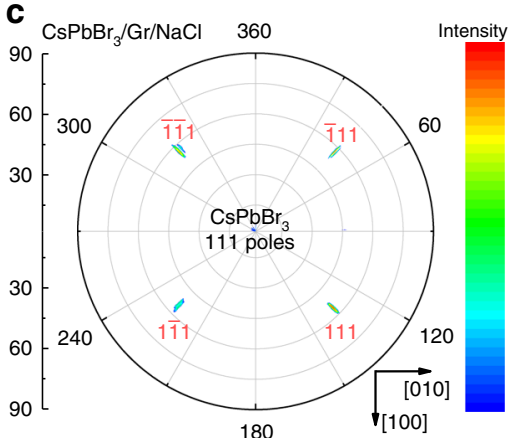

b

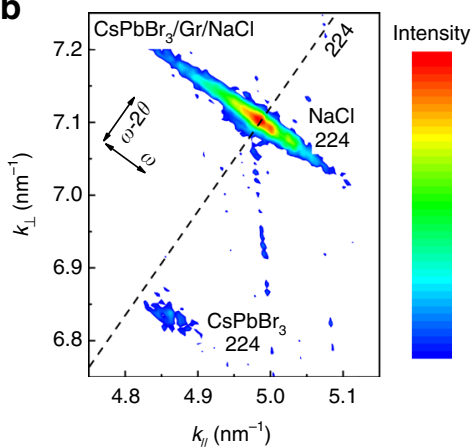

d

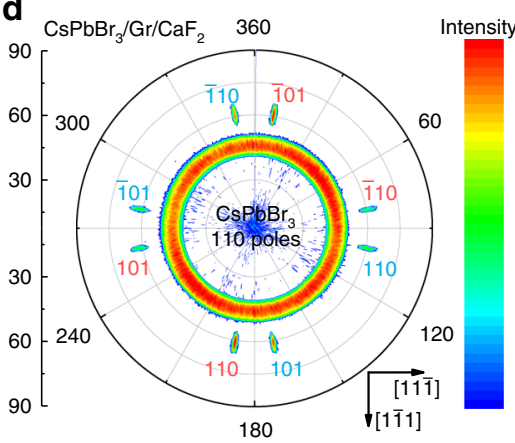

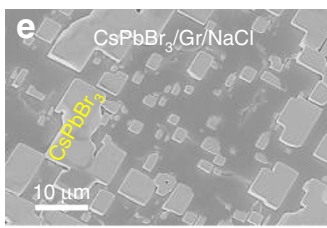
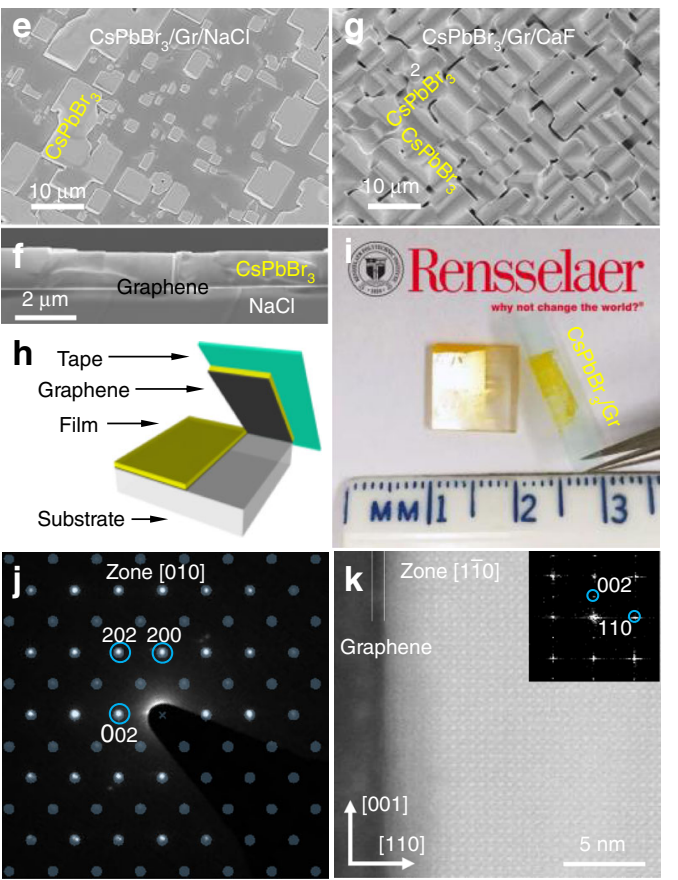

Fig. 1 Structure and morphology analysis of remote epitaxial $\mathrm{CsPbBr}_{3}$ a $\mathrm{RSM}$ of 224 peaks for $\mathrm{CsPbBr}_{3}$ thin film and $\mathrm{NaCl}$ from ionic epitaxy (CsPbBr $/$ $\mathrm{NaCl}$ ). b RSM of 224 peaks for $\mathrm{CsPbBr}_{3}$ flakes and $\mathrm{NaCl}$ from remote epitaxy $\left(\mathrm{CsPbBr}_{3} / \mathrm{Gr} / \mathrm{NaCl}\right.$ ). c X-ray pole figure of $\mathrm{CsPbBr} 3111 \mathrm{from} \mathrm{CsPbBr} / \mathrm{Gr}_{3} /$ $\mathrm{NaCl}$. d X-ray pole figure of $\mathrm{CsPbBr}_{3} 110$ from $\mathrm{CsPbBr}_{3} / \mathrm{Gr} / \mathrm{CaF}_{2}$. e SEM image of remote epitaxial $\mathrm{CsPbBr}_{3}$ flakes on $\mathrm{Gr} / \mathrm{NaCl}$. f Cross-sectional SEM image of the remote epitaxial $\mathrm{CsPbBr}_{3}$ thin film on $\mathrm{Gr} / \mathrm{NaCl}_{\mathbf{g}} \mathbf{g} \mathrm{SEM}$ image of remote epitaxial $\mathrm{CsPbBr}_{3}$ triangular prisms on $\mathrm{Gr} / \mathrm{CaF}$. $\mathbf{h}$ Schematic illustration of the exfoliation process on the remote epitaxial sample. $\mathbf{i}$ Photograph of the as-grown remote epitaxial thin film and exfoliated thin film. $\mathbf{j}$ Diffraction pattern of remote epitaxial $\mathrm{CsPbBr}_{3}$ (white dots) with simulated pattern on it (transparent bluish dots). $\mathbf{k}$ Cs-corrected STEM image of remote epitaxial CsPbBr 3 and FFT in the inset. (a-d are indexed by pseudocubic structure to simplify the analysis, $\mathbf{k}$ and $\mathbf{j}$ are indexed by $\mathrm{CsPbBr}_{3}$ orthorhombic structure)

roughness of $0.9 \mathrm{~nm}$ and $0.4 \mathrm{~nm}$ at $3 \mu \mathrm{m}$ lateral scale, respectively. The thickness of ionic and remote epitaxial flakes are estimated to be around $750 \mathrm{~nm}$ and $860 \mathrm{~nm}$ from the height profiles of Supplementary Fig. 6e, f, respectively. The surface morphology and height profiles of both ionic and remote epitaxial films are shown in Supplementary Fig. 6c, g and d, h, respectively. The surface RMS roughness for the ionic epitaxial film in Supplementary Fig. $6 \mathrm{c}$ is calculated to be around $4.6 \mathrm{~nm}$ at $10 \mu \mathrm{m}$ lateral scale, while it decreases to $1.5 \mathrm{~nm}$ for the remote epitaxial film in Supplementary Fig. 6d.

The as-grown $\mathrm{Cs} \mathrm{PbBr}_{3}$ flakes in remote epitaxy and the status of graphene after growth are characterized by Raman spectrum and mapping, as shown in Supplementary Fig. $7 \mathrm{~b}$ and $\mathrm{c}-\mathrm{f}$, respectively. The Raman mapping region $\left(10 \times 10 \mu \mathrm{m}^{2}\right)$ is indicated in green square in Supplementary Fig. 7a. The Raman peaks at around $68 \mathrm{~cm}^{-1}, 120 \mathrm{~cm}^{-1}$, and $307 \mathrm{~cm}^{-1}$ confirm the orthorhombic phase of $\mathrm{CsPbrr}_{3}{ }^{51}$, as shown in Supplementary Fig. 7b, c. After growth, the graphene G-band and 2D-band can still be seen in Raman spectra in Supplementary Fig. 7b. Besides, additional D-band of graphene can be found under $\mathrm{CsPbBr}_{3}$ flakes, which might be related to the disorder induced by epitaxial growth. Supplementary Fig. 7f shows a slightly shift towards high wavenumber in $2 \mathrm{D}$-band at the region under flakes, indicating compression in graphene due to epitaxial strain.

Besides the $\mathrm{NaCl}$ substrate, the remote epitaxy of $\mathrm{CsPbBr}_{3}$ has also been achieved on $\mathrm{CaF}_{2}(001)$ substrate $\left(\mathrm{CsPbr}_{3} / \mathrm{Gr} / \mathrm{CaF}_{2}\right)$, as indicated from the $\mathrm{X}$-ray pole figure of $\mathrm{CsPbBr}_{3} 110$ in Fig. $1 \mathrm{~d}$ and $\varphi$-scan of $\mathrm{CsPbBr}_{3} 110$ and $\mathrm{CaF}_{2} 111$ in Supplementary Fig. 8. SEM image of Fig. $1 \mathrm{~g}$ and optical image of Supplementary Fig. 9a show the morphology of the epitaxial triangular prisms, revealing two $90^{\circ}$-rotated domains epitaxially grown on
$\mathrm{Gr} / \mathrm{CaF}_{2}(001)$. The epitaxial relationship between two kinds of epitaxial domains and the substrate is characterized by $\mathrm{X}$-ray pole figure of $\mathrm{CsPbBr}_{3} 110$ and $\varphi$-scan of $\mathrm{CsPbBr}_{3} 110$ and $\mathrm{CaF}_{2} 111$, which is out-of-plane $\mathrm{CsPbBr}_{3}(011) \| \mathrm{CaF}_{2}(001)$ and in-plane $\mathrm{CsPbBr}_{3}[100] \| \mathrm{CaF}_{2}[010]$ or $\mathrm{CsPbBr}_{3}[0 \overline{1} 1] \| \mathrm{CaF}_{2}[010]$.

The as-grown epilayer can be easily exfoliated. The exfoliation process is shown in the schematic illustration in Fig. $1 \mathrm{~h}$ and the corresponding photograph is shown in Fig. 1i. The exfoliated sample can be transferred on to transmission electron microscopy (TEM) grid. TEM of halide perovskite materials is a challenge due to degradation under electron beam ${ }^{52}$. Before degradation, TEM diffraction pattern is obtained, as shown in Fig. 1j. The diffraction spots can be well indexed to support the orthorhombic structure, and the zone axis is calculated to be [010]. The cross-sectional interfacial morphologies and crystallite structures were evaluated by low-voltage Cs-corrected scanning transmission electron microscope (STEM). The STEM samples of $\mathrm{CsPbBr}_{3} / \mathrm{Gr} / \mathrm{NaCl}$ were prepared by focused ion beam (FIB) and details are shown in Methods. Figure $1 \mathrm{k}$ demonstrates smooth interfaces between $\mathrm{CsPbBr}_{3}$ (bright part), $\mathrm{NaCl}$ (dark part) and $\mathrm{Gr}$ (dark line in between) and atomic-resolution image of epilayer $\mathrm{CsPbBr}_{3}$ for $\mathrm{CsPbBr} / \mathrm{Gr} / \mathrm{NaCl}$. The FFT in the inset of Fig. $1 \mathrm{k}$ confirms the orthorhombic phase of $\mathrm{CsPbBr}_{3}$. More atomic-scale images of epilayer $\mathrm{CsPbBr}_{3}$ and their FFTs are shown in Supplementary Fig. $10 \mathrm{a}, \mathrm{b}$ and their insets, respectively. It is noted that $\mathrm{NaCl}$ in Fig. $1 \mathrm{k}$ is amorphous because $\mathrm{NaCl}$ is extremely unstable even under low-voltage electron beam, as shown in Supplementary Fig. $11 \mathrm{a}-\mathrm{c}$ with holes formed. Comprehensive study on stability of both $\mathrm{CsPBBr}_{3}$ and $\mathrm{NaCl}$ was carried out by in-situ TEM, as shown in Supplementary Fig. 12 for $\mathrm{CsPbBr}_{3} / \mathrm{Gr} / \mathrm{NaCl}$ and Supplementary Fig. 13 for $\mathrm{NaCl}$ in another region. The variation of spots in 
FFTs in the insets of Supplementary Figs. 12 and 13 indicate both $\mathrm{CsPbBr}$ and $\mathrm{NaCl}$ are unstable and $\mathrm{CsPbBr}_{3}$ is slightly better than $\mathrm{NaCl}$. Supplementary Movies 1, 2 and 3 show the detail amorphization processes from bright-field images of $\mathrm{Cs} \mathrm{PbBr}_{3} / \mathrm{Gr} /$ $\mathrm{NaCl}$ and $\mathrm{NaCl}$ and diffraction pattern of $\mathrm{NaCl}$, respectively.

Unscreened polar substrate electrostatic potential. To understand the physical mechanism for the experimental observation of remote epitaxy in halide perovskite, DFT calculations were performed firstly. For $\mathrm{CsPbBr}_{3}(001)$ growth on $\mathrm{Gr} / \mathrm{NaCl}(001)$, relaxed four layers of $\mathrm{CsPbBr}_{3}(001)$ lattice on $\mathrm{NaCl}(001)$ and monolayer graphene-coated $\mathrm{NaCl}(001)$ were chosen, as shown in Supplementary Fig. 14a, b. The interlayer distance between $\mathrm{CsPbBr}_{3}(001)$ and $\mathrm{NaCl}(001)$ is $3.0 \AA$. The interlayer distances between $\mathrm{CsPbBr}_{3}(001)$ and graphene, and between graphene and $\mathrm{NaCl}(001)$ are both $3.1 \AA$ A. Figure $2 \mathrm{a}, \mathrm{b}$ present the corresponding charge transfer distributions between $\mathrm{CsPbBr}_{3}(001)$ and $\mathrm{NaCl}$ (001), and between $\mathrm{CsPbBr}_{3}(001)$ and $\mathrm{NaCl}(001)$ with monolayer graphene insertion. Comparing Fig. 2a, b, we can observe that the insertion of graphene does not change the tendency of charge redistribution between $\mathrm{CsPbBr}_{3}(001)$ and $\mathrm{NaCl}(001)$. The characteristics of the interfacial interactions between $\mathrm{CsPbBr}_{3}(001)$ and $\mathrm{NaCl}(001)$, and between $\mathrm{CsPbBr}_{3}(001)$ and monolayer graphene buffered $\mathrm{NaCl}(001)$ are reflected by their respective interfacial interaction energies in Fig. 2c. The atomic stacking between top layer $\mathrm{NaCl}(001)$ and graphene is presented in Fig. $2 \mathrm{~d}$. The electrostatic potential distribution contributed by both $\mathrm{NaCl}$ and graphene on the surface is shown in Fig. 2e. The observed pattern of blue spots is consistent with $\mathrm{NaCl}(001)$ atomic stacking in Fig. 2d, implying the influence of $\mathrm{NaCl}(001)$ on the orientation of growth $\mathrm{CsPbBr}_{3}$.

For $\mathrm{CsPbBr}_{3}(011)$ growth on $\mathrm{Gr} / \mathrm{CaF}_{2}(001)$, the relaxed three layers of $\mathrm{CsPbBr}_{3}(011)$ lattice on monolayer graphene-coated $\mathrm{CaF}_{2}(001)$ was chosen, as shown in Supplementary Fig. 14c. The interlayer distances between $\mathrm{CsPbBr}_{3}(011)$ and graphene, and between graphene and $\mathrm{CaF}_{2}(001)$ are $2.75 \AA$ and $2.26 \AA$, respectively. Figure $2 \mathrm{f}$ presents the corresponding charge transfer distribution between $\mathrm{CsPbBr}_{3}(011)$ and $\mathrm{CaF}_{2}(001)$ with monolayer graphene intercalation. From Fig. $2 \mathrm{f}$ we can observe that the $\mathrm{CaF}_{2}(001)$ substrate influences the charge distribution of graphene and subsequently modulates the growth of $\mathrm{CsPbBr}_{3}(011)$. Then the interfacial interaction energy between $\mathrm{CsPbBr}(011)$ and monolayer graphene-coated $\mathrm{CaF}_{2}(001)$ was calculated as $-27.83 \mathrm{meV}^{-2}$, indicating the graphene intercalation could effectively reduce the interfacial strain. The atomic stacking between top layer $\mathrm{CaF}_{2}(001)$ and graphene is presented in Fig. $2 \mathrm{~g}$. The electrostatic potential distribution contributed by the $\mathrm{Ca}$ atoms on the surface directly above graphene, with a distance of $2.7 \AA$ is shown in Fig. 2h. In Fig. 2h, the observed pattern of blue spots is consistent with $\mathrm{CaF}_{2}(001)$ atomic stacking in Fig. $2 \mathrm{~g}$, implying the influence of $\mathrm{CaF}_{2}(001)$ on the orientation of growth $\mathrm{CsPbBr}_{3}(011)$.

Suppressing nucleation and promoting growth in remote epitaxy. Based on the calculated interfacial free energies in both ionic and remote epitaxy, a semi-quantitative epitaxial nucleation and growth model is proposed. The schematic illustration in Fig. 3a describes the atomistic nucleation process at the initial stage of an ionic epitaxy and a remote epitaxy. Having the monolayer graphene on the substrate (right side of Fig. 3a), the adsorption energy, $E_{\mathrm{ad}}$, and the diffusion energy, $E_{\mathrm{d}}$, are completely reduced, thereby affecting the adsorption and diffusion of atoms on the substrate surface. The weakened surface electrostatic potential after graphene screening and reduced adatom-substrate interaction in the case of remote epitaxy would lead to large critical nuclei, while the stronger ionic bond in ionic epitaxy leads to smaller nuclei, as sketched in purple adatoms in Fig. 3a. In addition, the extremely low diffusion barrier for adatoms on graphene in remote epitaxy makes the adatoms much easier to diffuse to the existing nuclei. Therefore, large size of nuclei with limited quantity is expected for remote epitaxy, as illustrated in Fig. 3a.

The nucleation rate could be calculated based on the classical nucleation theory. Assuming monolayer 2D disc-shaped nuclei at the initial state of growth, the Gibbs free energy of formation for nuclei is given by:

$$
\Delta G_{\mathrm{i}}=-\pi r^{2} a_{0} \Delta \mu+\pi r^{2}\left(\gamma_{\text {int }}+\gamma_{\mathrm{c}}-\gamma_{\mathrm{s}}\right)+2 \pi r a_{0} \gamma_{\mathrm{c}},
$$

where $a_{0}$ is taken to be the lattice constant of $\mathrm{CsPbBr}_{3}$ with respect to the ideal perovskite, $\Delta \mu$ is the difference in volumetric Gibbs free energy of the two phases (solid and gas) (unit: $\mathrm{meV}^{-3}$ ) and a general description of supersaturation for $\Delta \mu$ is used, $\gamma_{\text {int }}$ is the interfacial free energy $\left(29.0 \mathrm{meV}^{-2}\right.$ and $21.8 \mathrm{meV}^{-2}$ from DFT calculation for ionic and remote epitaxy as shown in Fig. 2c, respectively), $\gamma_{c}$ is the surface free energy of $\mathrm{Cs} \mathrm{PbBr}_{3}$ (76.3 $\mathrm{meV}^{-2}$, approximated from the bond energy of $248 \mathrm{KJ} \mathrm{mol}^{-1}$ for $\mathrm{Pb}-\mathrm{Br}$ and $389 \mathrm{KJ} \mathrm{mol}^{-1}$ for $\mathrm{Cs}-\mathrm{Br}^{53}$ ), $\gamma_{\mathrm{s}}$ is the surface free energy of substrate $\left(90.3 \mathrm{meV}^{-2}\right.$ for ionic epitaxy approximated from the bond energy of $412 \mathrm{KJ} \mathrm{mol}^{-1}$ for $\mathrm{Na}-\mathrm{Cl}^{53}$ and $2.9 \mathrm{meV}^{-2}$ for remote epitaxy based on the surface free energy of $48 \mathrm{~mJ} \mathrm{~m}^{-2}$ for graphene). The critical nucleus size $r^{*}$ can be obtained by maximizing $\Delta G_{\mathrm{i}}$ with respect to $r$, which is given by:

$$
r^{*}=\frac{\gamma_{\mathrm{c}}}{\Delta \mu-\left(\gamma_{\mathrm{int}}+\gamma_{\mathrm{c}}-\gamma_{\mathrm{s}}\right) / a_{0}} .
$$

Thus, the critical Gibbs free energy of formation for a monolayer 2D disc-shaped nucleus is given by:

$$
\Delta G_{\mathrm{i}}^{*}=\frac{\pi a \gamma_{\mathrm{c}}^{2}}{\Delta \mu-\left(\gamma_{\mathrm{int}}+\gamma_{\mathrm{c}}-\gamma_{\mathrm{s}}\right) / a_{0}}=\pi r^{*} a_{0} \gamma_{\mathrm{c}} .
$$

Hence, the nucleation rate can be expressed as:

$$
N=\Omega \exp \left[-\frac{\Delta G_{\mathrm{i}}^{*}}{k_{\mathrm{B}} T}\right]=\Omega \exp \left[-\frac{\pi a \gamma_{\mathrm{c}}^{2}}{k_{\mathrm{B}} T\left[\Delta \mu-\left(\gamma_{\mathrm{int}}+\gamma_{\mathrm{c}}-\gamma_{\mathrm{s}}\right) / a_{0}\right]}\right]
$$

where $\Omega$ is the pre-exponential factor, which is not very much dependent upon the supersaturation and a typical value of $10^{17} \mathrm{~cm}^{-2} \mathrm{~s}^{-1}$ is taken for condensation from vapor in our case, $k_{\mathrm{B}}$ is the Boltzmann constant, and $T$ is the temperature $(500 \mathrm{~K}$ was taken). The nucleation rates in both ionic and remote epitaxy are plotted as a function of supersaturation, as shown in Fig. 3b. The remote epitaxy shows orders of magnitude decrease in nucleation rate comparing to the ionic epitaxy and the decrease even reaches four orders of magnitude at low supersaturation.

Guided from our previous study on ionic epitaxy of halide perovskite ${ }^{54}$, the supersaturation for the epitaxial growth could be well controlled by the substrate temperature. In the present work, a temperature gradient on a single substrate has been introduced, leading a supersaturation gradient and different morphologies of halide perovskites on the substrate. Based on the nucleation rates predicted in Fig. 3b, a schematic illustration of a growth model of $\mathrm{Cs} \mathrm{PbBr}_{3}$ epitaxially grown on $\mathrm{NaCl}$ substrate with graphene covered half area is presented in Fig. 3c. For ionic epitaxy, high supersaturation leads the growth of epitaxial thin film, while low supersaturation leads the growth of epitaxial flakes. For remote epitaxy, at the same supersaturation, the nuclei are much sparser than that in epitaxial thin film for the ionic epitaxy, resulting in the growth of epitaxial flakes. The SEM images in Fig. 3d-h and the optical microscope images in Supplementary Fig. 9b, c show 

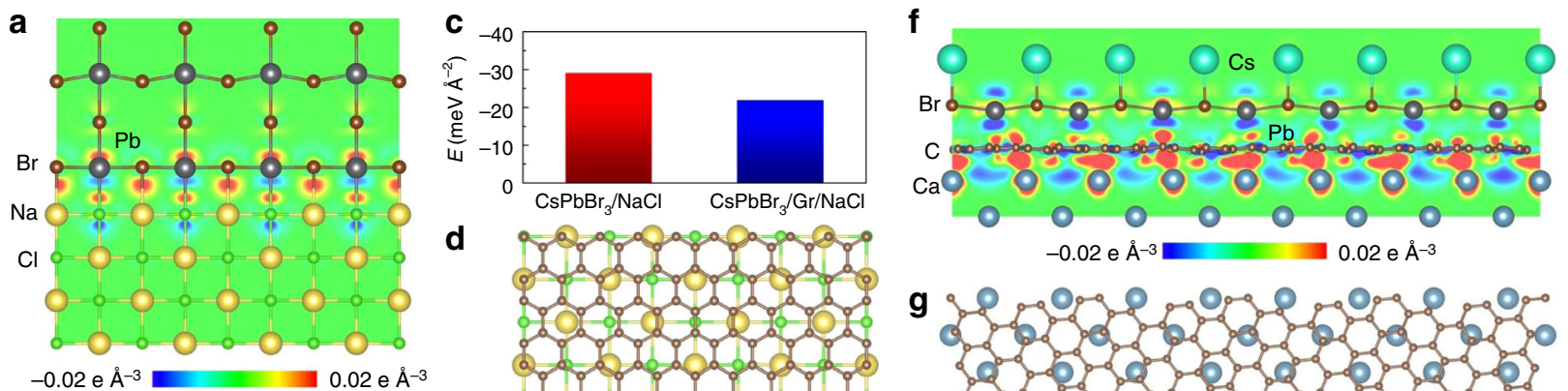

d
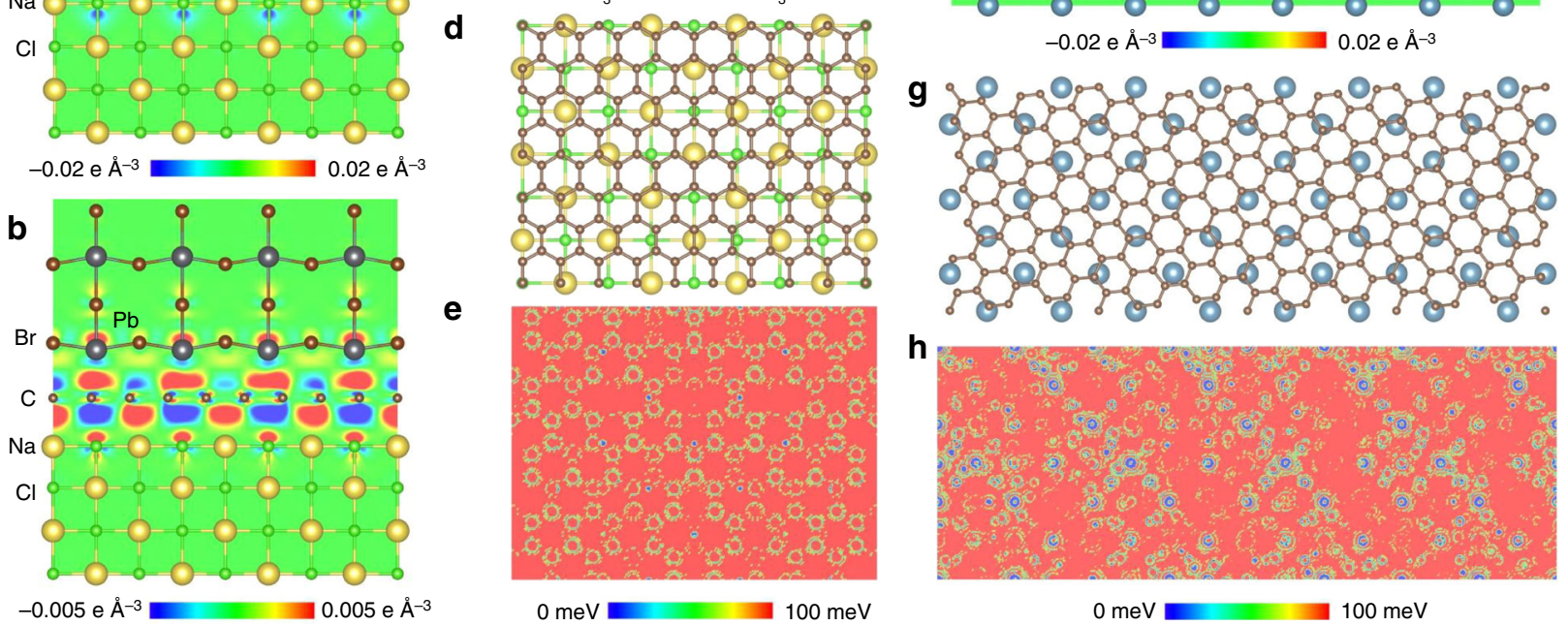

$0 \mathrm{meV} 100 \mathrm{meV}$

Fig. 2 lonic and remote atomic interactions between polar substrates and $\mathrm{CsPBrr}_{3} . \mathbf{a}, \mathbf{b}$ Charge transfer distributions between $\mathrm{CsPbBr}(001)$ and $\mathrm{NaCl}_{3}$ (001) (a), and between $\mathrm{CsPbBr}_{3}(001)$ and $\mathrm{NaCl}(001)$ with graphene intercalation (b). c Interfacial interactions between $\mathrm{CsPbBr}_{3}(001)$ and $\mathrm{NaCl}(001)$, between $\mathrm{CsPbr}_{3}(001)$ and monolayer graphene buffered $\mathrm{NaCl}(001)$. d Atomic stacking between $\mathrm{NaCl}(001)$ top layer and buffered monolayer graphene. e Potential fluctuation at the epitaxial surface from $\mathrm{NaCl}(001)$ through monolayer graphene, where blue pattern is consistent with atomic stacking of $\mathrm{NaCl}$ (001) in (d). $\mathbf{f}$ Charge transfer distribution between $\mathrm{CsPbBr}_{3}(011)$ and $\mathrm{CaF}_{2}(001)$ with graphene intercalation. $\mathbf{g}$ Atomic stacking between $\mathrm{CaF}$ (001) top layer and coated monolayer graphene. $\mathbf{h}$ Potential fluctuation at the epitaxial surface from $\mathrm{CaF}_{2}(001)$ through monolayer graphene, where blue pattern is consistent with center site of four Ca atoms in (g)

the various morphologies of specific interesting areas on a typical sample, which are ionic epitaxial thin film at high supersaturation in Fig. 3d and left side of $3 \mathrm{e}$, ionic epitaxial flakes at low supersaturation in Supplementary Fig. 9b, remote epitaxial thin film and flakes at high supersaturation in right side of Fig. 3e, f, respectively, remote epitaxial flakes at low supersaturation in Fig. 3g, h and Supplementary Fig. 9c. These morphologies are well consistent with the theoretical model in Fig. 3b. By counting the number of flakes at the region shown in Fig. 1e for the remote epitaxy case, with the assumption of constant nucleation rate during the growth and the growth time of 15 minutes for the sample of Fig. 3g, the nucleation rate is estimated to be around $4.4 \times 10^{5} \mathrm{~cm}^{-2} \mathrm{~s}^{-1}$.

Dislocation formation in ionic and remote epitaxy. Dislocations are often harmful to device performance. Under thermodynamically favorable condition, dislocation-free crystals are accessible when the growth of crystals from solutions is at low supersaturation or from melts is weakly supercooled ${ }^{55}$. However, dislocation formation is thermodynamically favored in highly mismatched heteroepitaxy system if the film thickness is above its critical thickness (in order to relax the misfit stain energy in epilayer ${ }^{56}$ ). Traditionally, a buffer layer can be introduced into epitaxial growth to reduce dislocation density ${ }^{57}$. By using a special buffer layer of graphene to reduce the interfacial energy between epilayer and substrate, the epitaxial growth mechanism (thermodynamics and kinetics) in remote epitaxy is significantly different from that in traditional epitaxy. Thus, different strain relaxation mechanism is expected. Molecular-dynamics (MD) simulations are utilized to study the growth and strain relaxation mechanisms in ionic epitaxy, remote epitaxy and van der Waals epitaxy. Figure $4 \mathrm{a}, \mathrm{d}$ and g shows MD simulations of the side views of ionic epitaxy, remote epitaxy and van der Waals epitaxy, respectively. The dislocations are indicated by blue arrows, as shown in Fig. 4a-c. Supplementary Fig. 15a and Supplementary Fig. 16 show the side and top views of the formation of dislocation in ionic epitaxy, respectively. Surprisingly, no dislocation can be formed in both remote and van der Waals epitaxy. In order to release strain energy between epilayer and substrate during the growth, large interfacial energy in ionic epitaxy enables the formation of dislocations. Misfit dislocations at the interface are observed in the side view in Supplementary Fig. 15a and threading dislocations are observed in top views in Supplementary Fig. 16. For both remote and van der Waals epitaxy, small interfacial energy enables small crystal islands gliding to big ones. The gliding of crystal islands is indicated by arrows in the side view of Supplementary Fig. 15b (remote epitaxy), top views of Supplementary Fig. 17 (remote epitaxy) and Supplementary Fig. 18 (van der Waals epitaxy). Top views of Supplementary Fig. 1618 also show the decrease of nucleation sites and the ability of gliding with decreasing interfacial energy (from ionic epitaxy, remote epitaxy to van der Waals epitaxy). Supplementary Movie 4, 5 and 6 show the side views of the entire growth processes of ionic epitaxy, remote epitaxy and van der Waals epitaxy, respectively. Supplementary Movie 7, 8 and 9 are the top views of ionic epitaxy, remote epitaxy and van der Waals epitaxy, respectively.

STEM, HRAFM, and HRTEM were utilized to characterize an as-grown remote epitaxial sample $\mathrm{CsPbBr}_{3} / \mathrm{Gr} / \mathrm{NaCl}$. Perfect crystallite atomic structures of $\mathrm{CsPbBr}_{3}$ are shown in cross-sectional images of Fig. 1k, Supplementary Fig. 10a, b, and Fig. 4j, k obtained by Cs-corrected STEM. FFTs in their insets confirm the orthorhombic phase of $\mathrm{CsPbBr}_{3}$. Two ferroelastic domains with zone axes of [001] and [110], as shown in Fig. 4j, k, respectively, are consistent with two out-of-plane peaks in the XRD $\omega-2 \theta$ scan in Supplementary Fig. 3. Supplementary Fig. 10c 
a

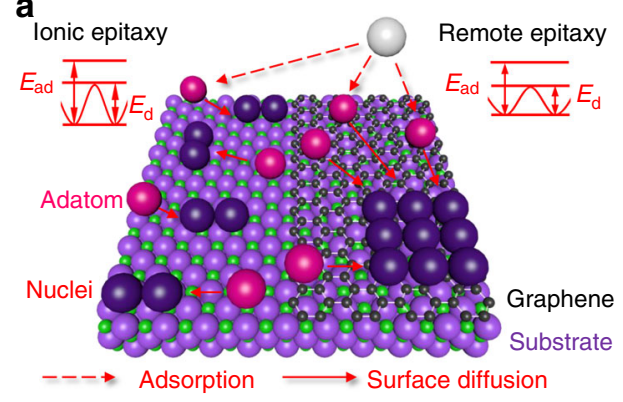

b

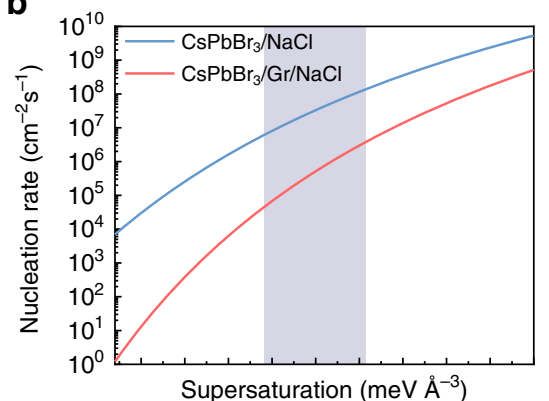

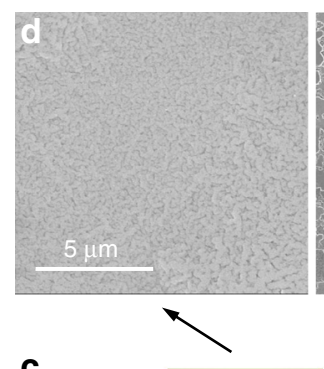
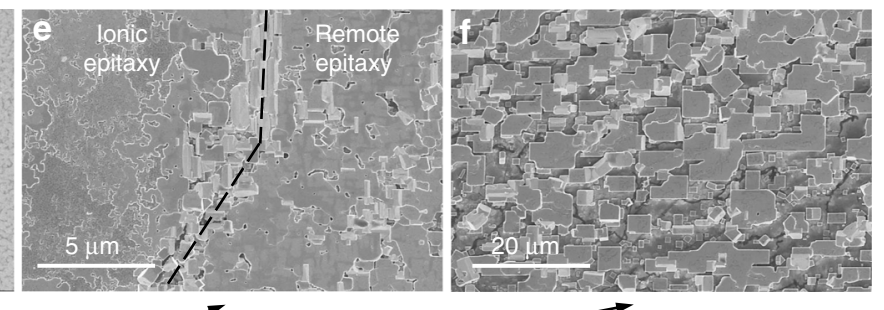

C
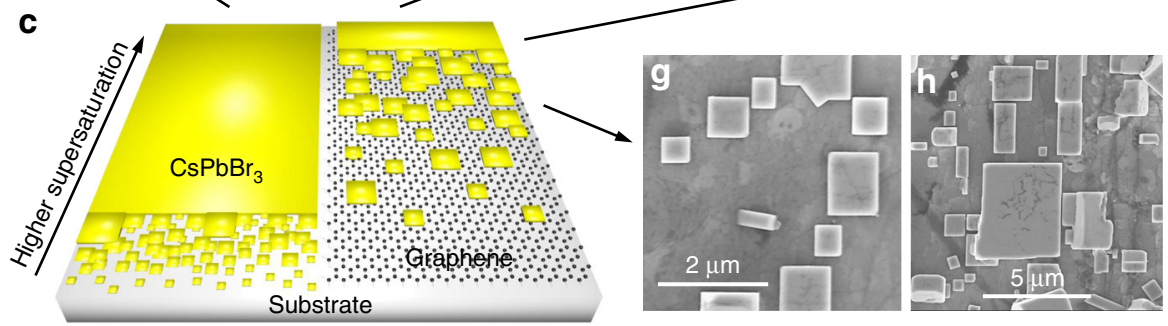

Fig. 3 Growth kinetics of ionic and remote epitaxy. a Schematic illustration of atomistic nucleation process at the initial stages of both ionic and remote epitaxy. $\mathbf{b}$ Nucleation rate as a function of supersaturation for both ionic epitaxy $\left(\mathrm{CsPbBr}_{3} / \mathrm{NaCl}\right)$ and remote epitaxy $\left(\mathrm{CsPbBr} / \mathrm{Gr}_{3} / \mathrm{NaCl}\right)$. c Schematic illustration of the nucleation process at the final stages of both ionic and remote epitaxy. $\mathbf{d}$-h $\mathrm{SEM}$ images of specific interesting regions on a typical sample denoted by black arrow

(with zoom-in part shown in Fig. $4 \mathrm{~m}$ ) and Supplementary Fig. 10d (with zoom-in part shown in Fig. $4 \mathrm{n}$ ) are inverse FFTs of their insets with highlighted white spots to highlight lattice fringes from Supplementary Fig. 10b, indicating no misfit dislocation. The atomic-scale image of a surface region of remote epitaxial film obtained from HRAFM exhibits perfect crystallite structure as well, as shown in Supplementary Fig. 19a. Figure 41 and Supplementary Fig. 19b are the inverse FFTs of their insets to highlight atomic structure fringes from Supplementary Fig. 10b and Supplementary Fig. 19a, indicating no threading dislocation. However, after carefully searching of many images, threading dislocations can be observed in remote epitaxial $\mathrm{CsPbBr}$ from some HRAFM and HRTEM images, as shown in highlighted lattice fringes in Supplementary Fig. 19d, Fig. 4o and Supplementary Fig. 20d-f (inverse FFTs of their insets transformed from Supplementary Figs. 19c, 20c and 20a-c, respectively). These threading dislocations observed from the remote epitaxial sample might be formed during large grain coalescence stage. More threading dislocations are found at a surface region of ionic epitaxial film, as shown in Supplementary Fig. 21d (inverse FFT of its inset transformed from Supplementary Fig. 21c), while similar perfect crystallite lattice is found at another surface region, as shown in Supplementary Fig. 21b (inverse FFT of its inset transformed from Supplementary Fig. 21a). It is noted that the density of dislocations could not be estimated from these atomicscale images due to low density and uneven distribution of dislocations.

van der Waals epitaxy of halide perovskite on a native oxide $\mathrm{Si}$ (100) substrate (non-polar) with transferred graphene on its surface $(\mathrm{Gr} / \mathrm{Si}(100))$ has been demonstrated in Supplementary
Fig. 22a. As expected, remote epitaxy shows better epitaxy and higher nucleation rate than van der Waals epitaxy with $\mathrm{Gr} / \mathrm{Si}$ (100) as substrate. This highlights the importance of extra potential guide of substrate in remote epitaxy. To improve substrate interaction, mica is chosen to substitute the $\mathrm{Gr} / \mathrm{Si}(100)$ substrate. As shown in Supplementary Fig. 20b, c, better crystal quality of halide perovskite (compared to $\mathrm{Gr} / \mathrm{Si}$ ) has been achieved in our growth. However, due to the in-plane symmetry mismatch between halide perovskite and mica, the epitaxial halide perovskite flakes show in-plane rotation (Supplementary Fig. 22d). Without the guide of potential field with matched symmetry from the polar substrate, the in-plane film quality in such quasi-van der Waals epitaxy is lower than that in remote epitaxy. However, the out-of-plane crystallinity of halide perovskite is better compared to that for remote epitaxy, as shown in XRD $\theta-2 \theta$ scanning and rocking curve in Supplementary Fig. 20d, f, respectively.

Additionally, the interfacial energy between substrate and epilayer could control the ferroelastic phase transition kinetics. Ionic epitaxial $\mathrm{CsPbBr}_{3}$ shows small spatial periodicity of the ferroelastic domains, while remote epitaxial one shows large spatial periodicity of the ferroelastic domains. Detailed results and discussion of controlling domain wavelength via remote epitaxy are presented in Supplementary Note 1 and Supplementary Figs. 26 and 27.

Misfit and threading dislocation-carrier dynamics relation. According to Matthew's theory, one could evaluate the lower limit of both misfit and threading dislocations densities in 

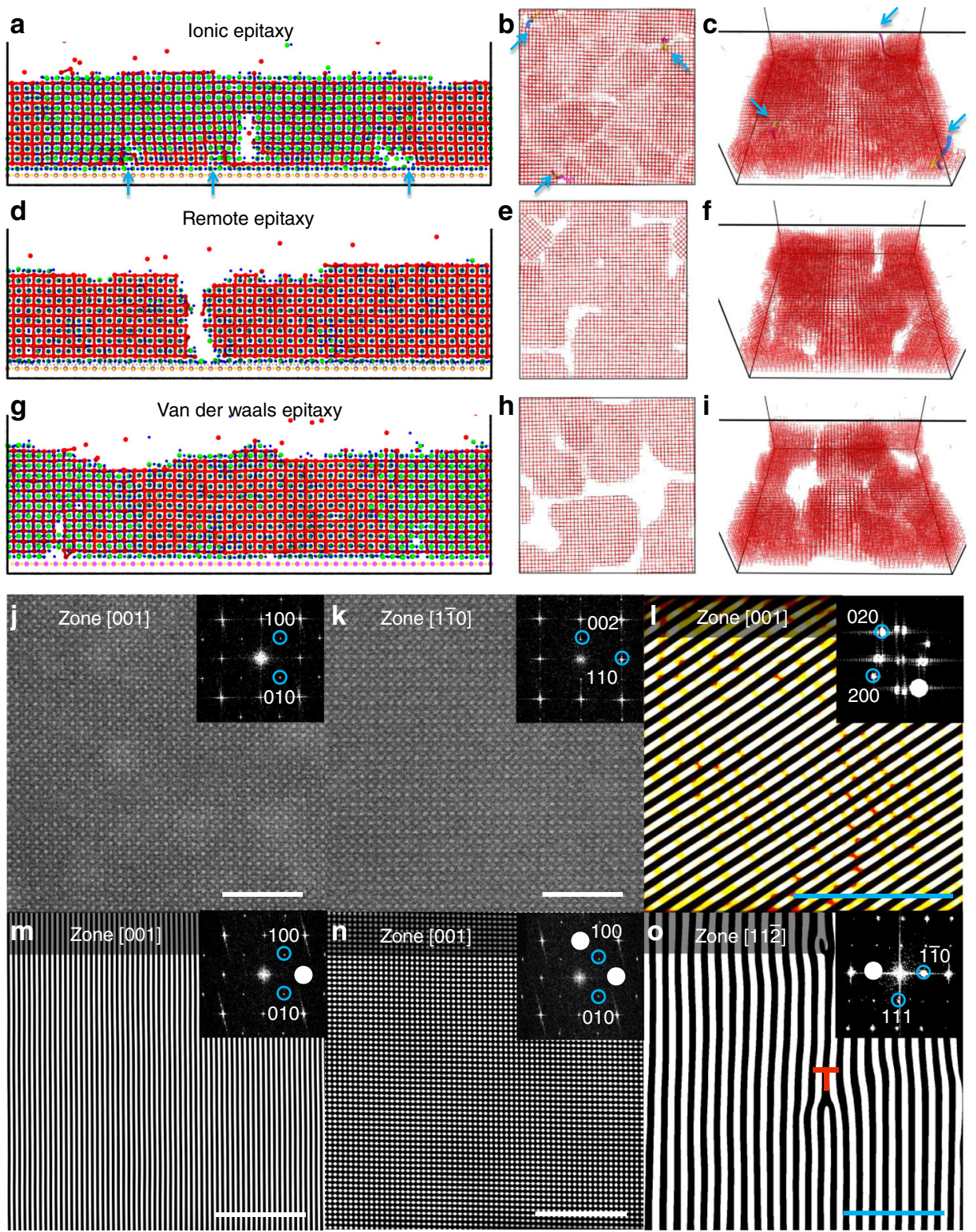

Fig. 4 Molecular-dynamics simulations of ionic epitaxy (a-c), remote epitaxy (d-f) and vdW epitaxy (g-i). $\mathbf{a}, \mathbf{d}$ and $\mathbf{g}$ are side views. $\mathbf{b}, \mathbf{e}$ and $\mathbf{h}$ are top views. $\mathbf{c}, \mathbf{f}$ and $\mathbf{i}$ are bird views. Dislocations are indicated by blue arrows. $\mathbf{j}, \mathbf{k}$ STEM images for different domains of $\mathrm{CsPbBr} / \mathrm{Gr} / \mathrm{NaCl}$ with zone axes of [001] (j) and [110] (k) and FFTs in their insets. I Inverse FFT of its inset transformed from the HRAFM image (Supplementary Fig. 19a) for CsPbBr $3 / \mathrm{Gr} /$ $\mathrm{NaCl}$. $\mathbf{m}, \mathbf{n}$, Zoom-in inverse FFTs of their insets transformed from the STEM images Supplementary Fig. $10 a$, b for $\mathrm{CsPbBr} / \mathrm{Gr} / \mathrm{NaCl}$, respectively. - Inverse FFT of its inset transformed from the HRTEM image (Supplementary Fig. 21c) for transferred $\mathrm{CsPbBr}_{3}$. A dislocation is indicated in o. Scale bars of $\mathbf{j}, \mathbf{k}, \mathbf{l}, \mathbf{o}$ are $5 \mathrm{~nm}$. Scale bars of $\mathbf{m}, \mathbf{n}$ are $10 \mathrm{~nm}$

epitaxial film when lattice mismatch and film-substrate interaction strength are given. Clearly, remote epitaxial film is expected to take much less dislocations than ionic epitaxial film. To understand the impact of dislocations on the carrier dynamics of halide perovskite, it is natural to compare the optoelectronic properties in these two types of films. The optical properties of a typical sample have been studied by steady-state photoluminescence (PL) measurements firstly, as shown in Fig. 5a, b. An intensive green light can be seen from the optical microscopy image in the inset of Fig. 5b, due to the band emission. Remarkable enhancements of around 3.7 and 2 times in the PL intensity can be clearly observed in both $\mathrm{CsPbBr}_{3}$ thin film and flake from remote epitaxy comparing to these from ionic epitaxy, respectively.
The optical properties of the as-grown $\mathrm{CsPbBr}_{3}$ thin films and flakes from both remote epitaxy and ionic epitaxy have been further investigated by time-resolved photoluminescence (TRPL) spectroscopy. The schematic illustration in Fig. $5 \mathrm{c}$ describes the carrier dynamics in semiconductor thin film, e.g. halide perovskite in the present case, including the effects of dislocations. The observed PL recombination dynamics could include contributions from Shockley-Read-Hall processes, radiative recombination, Auger recombination, and interface recombination ${ }^{58,59}$. Misfit and threading dislocations can be formed above the critical thickness of the film in semiconductors, e.g. $\mathrm{GaP}^{60}, \mathrm{GaN}^{4}$, inducing defect trap states, as shown in Fig. $5 \mathrm{c}$, thereby influence or even determine the effective carrier lifetime via non-radiative recombination. By taking account of the grain 
a

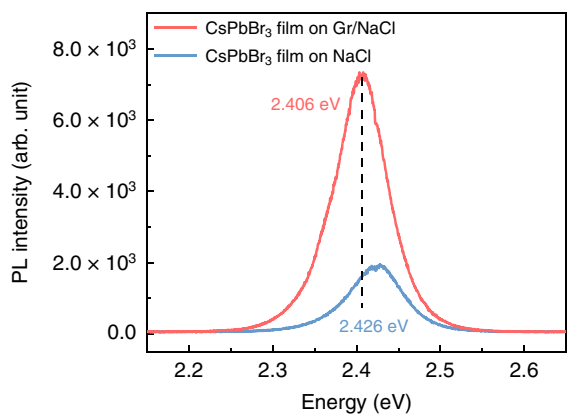

d

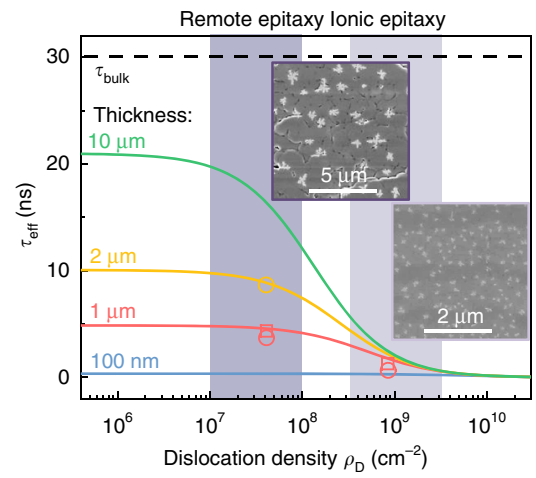

b

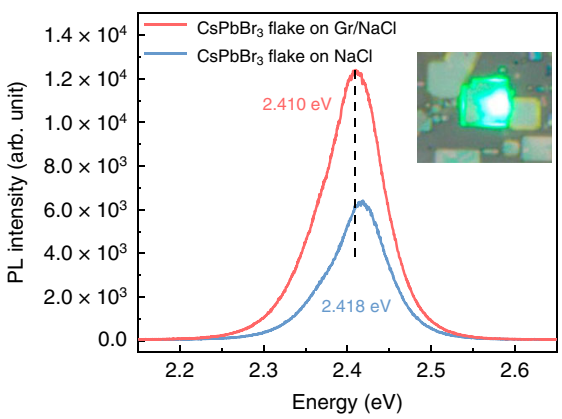

e

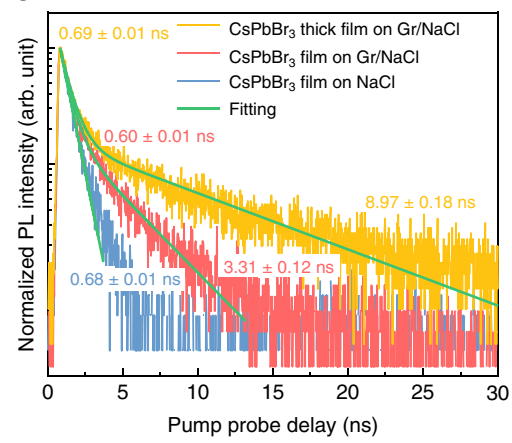

C

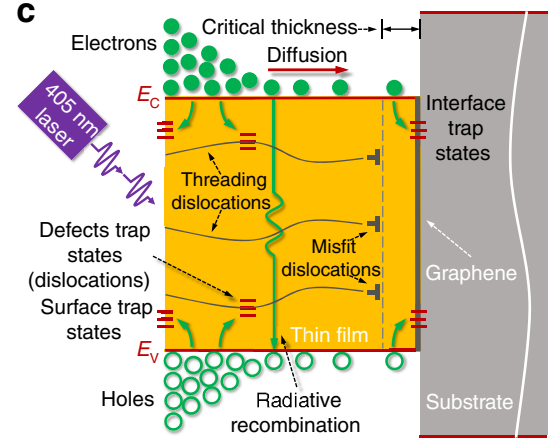

$\mathbf{f}$

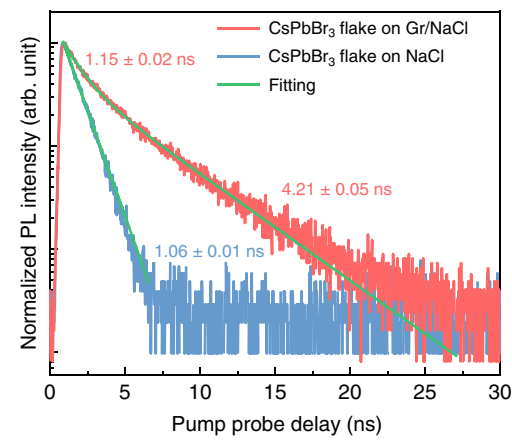

Fig. 5 Dislocation-carrier dynamics relation in remote and ionic epitaxy. $\mathbf{a}$, b, Steady-state $\mathrm{PL}$ of $\mathrm{CsPbBr}_{3}$ films (a) and flakes (b) in both remote and ionic epitaxy. c Schematic illustration of carrier dynamics in $\mathrm{CsPbBr}_{3}$. d Effective carrier lifetime as a function of dislocation density with different sample thickness and SEM images for ionic (right one) and remote (left one) epitaxial samples after etching. Experimental data for thin film and flake are indicated in circle and square, respectively. Remote epitaxy and ionic epitaxy regions painted in dark purple and light purple, respectively. e, $\mathbf{f}, \mathrm{TRPL}_{\mathrm{r}}$ of $\mathrm{CsPbBr}{ }_{3}$ films (e) and flakes (f) in both remote and ionic epitaxy

boundary recombination and dislocation recombination, with the assumption of same diffusivity of the excess carriers as the minority carriers at low injection and no junction on an array of dislocations in an infinite semiconductor, the effective lifetime can be modified from the previous works by refs. $28,54,61,62$

$$
\begin{array}{r}
\frac{1}{\tau_{\text {eff }}}=\frac{1}{\tau_{\text {bulk }}}+\frac{1}{\tau_{\text {int }}}+\frac{1}{\tau_{\mathrm{gb}}}+\frac{1}{\tau_{\text {dis }}}=\frac{1}{\tau_{\text {bulk }}} \\
+\frac{1}{\frac{t}{2 S_{\text {int }}}+\frac{1}{D}\left(\frac{t}{\pi}\right)^{2}}+\frac{1}{\frac{d}{2 S_{\mathrm{gb}}}+\frac{1}{D}\left(\frac{d}{\pi}\right)^{2}}+\frac{4 \pi D \rho_{\mathrm{D}}}{-\ln \left(\pi \rho_{\mathrm{D}} r_{0}^{2}\right)-\frac{6}{5}}
\end{array}
$$

where $\tau_{\mathrm{gb}}$ is the grain boundary carrier lifetime, $\tau_{\text {dis }}$ is the dislocation carrier lifetime, $S_{\mathrm{int}}$ and $S_{\mathrm{gb}}$ is the recombination velocity at the interface and grain boundary, respectively, $d$ is the lateral grain size, $t$ is the vertical thickness, $D$ is the diffusivity, $\rho_{\mathrm{D}}$ is the threading dislocation density, and $r_{0}$ is the effective core radius of a dislocation inside which the lifetime is zero. The recombination velocity at interface $S_{\text {int }}$ is influenced by the growth condition and interfacial chemistry, while the recombination velocity at grain boundary $S_{\mathrm{gb}}$ is fixed for the same material. Here we assume the recombination velocity at both interface and grain boundary are the same $\left(1.5 \times 10^{4} \mathrm{~cm} \mathrm{~s}^{-1}\right.$ was taken $\left.{ }^{63}\right)$ and focus on the dislocation recombination effect. The diffusivity $D$ is taken to be $0.35 \mathrm{~cm}^{2} \mathrm{~s}^{-163}, r_{0}$ is taken to be $5.87 \times 10^{-8} \mathrm{~cm}$ (the lattice constant of cubic $\mathrm{Cs} \mathrm{PbBr}_{3}$ ). The lateral grain size $d$ of around $1-5 \mu \mathrm{m}$ was roughly measured by the SEM images Fig. 3 . By using equation (5) with constant values of sample thickness and grain size, the effective lifetime of halide perovskite $\mathrm{Cs} \mathrm{PbBr}_{3}$ is plotted as a function of dislocation density, as shown in Fig. $5 \mathrm{~d}$ and Supplementary Fig. 23 for different sample thickness and grain size, respectively. Thus, the effect of dislocation density on the effective lifetime of halide perovskite $\mathrm{CsPbr}_{3}$ is quantitatively analyzed. The thickness and the grain size can influence the effective lifetime as well, as shown in lines with different colors in Fig. 5d and Supplementary Fig. 23, respectively. Obviously, the effective lifetime reduces drastically with increasing dislocation density if we assume dislocations in halide perovskite provide recombination centers.

To experimental show whether the dislocations contribute significant recombination centers or not, we have further quantified the dislocation density and correlated them with the TRPL lifetime. The threading dislocation density can be estimated by dislocation etch pits. The etching technique for $\mathrm{CsPbBr}_{3}$ is similar to that for $\mathrm{NaCl}^{64}$, but with different concentration of etching solution. Dislocation etch rosettes have been formed on the surface of the remote epitaxial sample, ionic epitaxial sample and $\mathrm{NaCl}$ substrate for comparison, as shown in the left and right inset of Fig. $5 \mathrm{~d}$ and Supplementary Fig. 5d. From the density of dislocation etch rosettes, for the ionic epitaxy, the threading dislocation density is estimated to be around $9 \times 10^{8} \mathrm{~cm}^{-2}$, while for the remote epitaxy, it is remarkably reduced to around $3 \times 10^{7} \mathrm{~cm}^{-2}$. Both remote epitaxy and ionic epitaxy regions are painted in dark gray and light gray in Fig. 5d, respectively.

The effective lifetimes have been extracted from the measured TRPL spectra for both $\mathrm{CsPbBr}_{3}$ thin film and flake in both remote and ionic epitaxy in Fig. 5e, f, respectively. Two kinds of recombination regimes can be extracted, including the short carrier lifetime dominated by the interface recombination and the long carrier lifetime dominated by the bulk recombination. The grain boundary and dislocation recombination can affect both regimes' carrier lifetime. For the remote epitaxial thin film comparing to the ionic epitaxial one, the effective lifetime of longlife carrier is greatly increased from 0.68 to $3.31 \mathrm{~ns}$, while for the 
remote epitaxial flake it increases from 1.06 to $4.21 \mathrm{~ns}$. The significant prolonged carrier lifetime of about four times in magnitude could be attributed to the more than one order of magnitude decrease in threading dislocation density. With doubled growth time, thick film shows effective lifetime up to $8.97 \mathrm{~ns}$, as shown in Fig. 5e. In single flakes with much smaller lateral size comparing to the thin film, the carrier lifetimes, as shown in Fig. 5f, however, are slightly longer than these in films, as shown in Fig. 5e. The extracted carrier lifetimes are good consistent with the hypothesis in Fig. 5d, indicating the predominant factor of dislocation recombination for the carrier dynamics in halide perovskite $\mathrm{CsPbBr}_{3}$ and the remarkable improvement of carrier lifetime in remote epitaxy. Similar enhancement of the effective lifetime is also observed in the case of $\mathrm{CsPbBr}_{3}$ grown on $\mathrm{Gr} / \mathrm{CaF}_{2}$, as shown in Supplementary Fig. 24 . These evidence clearly show that threading dislocation is as important as grain boundary or point defect in halide perovskite for physical properties and device performance.

Two gold stripe contacts are deposited onto the $\mathrm{CsPbBr}_{3}$ thin film with a physical mask on top by e-beam evaporation and a simple device of photodetector $\left(\mathrm{Au} / \mathrm{CsPbBr}_{3}\right.$ (remote epitaxial thin film)/Au) is fabricated, as shown in Supplementary Fig. 25a. The photo responses under UV light $(405 \mathrm{~nm}$ laser, around $0.5 \mathrm{~mW}$ ) are measured for both devices made by ionic and remote epitaxial thin films, as shown in Supplementary Fig. 25b. Supplementary Fig. 25c, d are the enlarged rising and falling parts in Supplementary Fig. 25b, respectively, revealing a faster rise time in remote epitaxial thin film-based device (Supplementary Fig. 25c) and an additional decay tail with a long decay time of around $8.08 \mathrm{~s}$ in ionic epitaxial thin film-based device (Supplementary Fig. 25d). These observations, i.e. fast rise time and disappearance of longer decay tail in remote epitaxial thin film-based device, could be attributed to the low density of dislocations in remote epitaxial thin film. Similar observations can be found in other photodetectors, such as $\mathrm{AlGaN}^{65}$.

\section{Discussion}

Epitaxial halide perovskite has been synthesized via a remote epitaxy approach. DFT simulations and experimental implementation show that the modified electrostatic potential of polar substrates coated with graphene enables tunable film-substrate interaction strength leading to controlled dislocation density in epilayer. The epitaxial relationships have been revealed by high resolution XRD RSMs and pole figures, which are out-of-plane $\mathrm{CsPbBr}_{3}(001) \| \mathrm{Gr} / \mathrm{NaCl}(001)$ and in-plane $\mathrm{CsPbBr}_{3}[100] \| \mathrm{Gr} / \mathrm{NaCl}[100]$ for remote epitaxy of $\mathrm{CsPbBr}_{3}$ on $\mathrm{Gr} / \mathrm{NaCl}$ and out-of-plane $\mathrm{CsPbBr}_{3}(011) \| \mathrm{CaF}_{2}(001)$ and in-plane $\mathrm{CsPbBr}_{3}[100] \| \mathrm{CaF}_{2}[010]$ or $\mathrm{CsPbBr}_{3}[011] \| \mathrm{CaF}_{2}[010]$ for remote epitaxy of $\mathrm{CsPBr}_{3}$ on $\mathrm{Gr} / \mathrm{CaF}_{2}$. The weak film-substrate coupling in remote epitaxy suppresses the nucleation and promotes the growth, and a semi-quantitative epitaxial nucleation and growth model is proposed to understand the growth kinetics. Further, the ferroelastic phase transition kinetics can be controlled by filmsubstrate coupling and remote epitaxy yields large spatial periodicity of the ferroelastic domains in $\mathrm{CsPbBr}_{3}$. In the cases of remote and van der Waals epitaxy with low interfacial energy, the dislocationfree or low-density dislocation mechanism is demonstrated by molecular-dynamics simulations. HRTEM, HRAFM and Cscorrected STEM studies show the atomic and lattice structure of the remote epitaxial films with very low-density threading dislocations. The controlling of dislocation (threading) density, which is estimated from etch rosettes, enables the unveiling of the dislocation-carrier dynamic relation in halide perovskite. Comparing to ionic epitaxy, remote epitaxy improves the PL intensity by around 3 times and the carrier lifetime by around 4 times in magnitude, indicating that the dislocation recombination is one of the predominant components for the carrier recombination mechanisms of halide perovskite. Our study reveals dislocation as another critical defect on influencing halide perovskite's optoelectronic properties in addition to grain boundary, point defects and phase impurity, and calls for further investigations on their control to tune halide perovskite device performances.

\section{Methods}

Graphene transfer. The graphene used in this work was purchased from Graphene Laboratories Inc. (Calverton, New York, US). The graphene was synthesized on $\mathrm{Cu}$ foils by chemical vapor deposition. For transfer of graphene, poly(methyl methacrylate) (PMMA) was spin-coated and baked on graphene/Cu to protect the graphene on the front side of $\mathrm{Cu}$ foils. The rear side of $\mathrm{Cu}$ foils was then treated in $\mathrm{O}_{2}$ plasma etcher to remove unwanted graphene. Afterward, the PMMA/graphene/ $\mathrm{Cu}$ stack was placed in ammonium persulfate aqueous solution $\left(60 \mathrm{~g} \mathrm{~L}^{-1}\right)$ to etch $\mathrm{Cu}$. Once $\mathrm{Cu}$ was etched, the PMMA/graphene was rinsed in water (isopropyl alcohol) several times and then scooped out using the $\mathrm{CaF}_{2}(\mathrm{NaCl})$ substrate. The PMMA/graphene/ $\mathrm{CaF}_{2}(\mathrm{NaCl})$ was dried in air. In the end, PMMA was dissolved in acetone.

\section{Remote epitaxy and ionic epitaxy of $\mathrm{CsPbBr}_{3}$ on $\mathrm{NaCl}$ and $\mathrm{CaF}_{2}$ substrates.} Cesium bromide (CsBr, 99\%, Sigma-Aldrich) was placed in the furnace heating center and lead(II) bromide $\left(\mathrm{PbBr}_{2}, 99 \%\right.$, Sigma-Aldrich) was placed about $10 \mathrm{~cm}$ away from the cesium bromide. The heating temperature was controlled at about $500^{\circ} \mathrm{C}$. Argon flow of $200 \mathrm{sccm}$ was introduced to the system to maintain the pressure at about 1.4 Torr during the growth. The growth process lasted for $15 \mathrm{~min}$. The details can be found in our previous report ${ }^{54}$. Prior to a growth, the monolayer graphene was transferred onto the substrates. The details on the transfer process can be found in our previous report ${ }^{66}$.

Dislocation etching technique. A solution of $\mathrm{FeCl}_{3}$ in glacial acetic acid was used for as-grown $\mathrm{CsPBBr}_{3}$ and substrate $\mathrm{NaCl}$ for comparison. Dislocation etch rosettes were obtained for both $\mathrm{NaCl}$ and $\mathrm{CsPBr}_{3}$ when they were moderately agitated for $60 \mathrm{~s}$ in $2.5 \times 10^{-2} \mathrm{~mol} \mathrm{~L}^{-1}$ and $2.5 \times 10^{-4} \mathrm{~mol} \mathrm{~L}^{-1} \mathrm{FeCl}_{3}$ in glacial acetic acid, respectively. After that, the samples were rinsed in acetone and dried in compressed air.

Focused ion beam (FIB). The lamellar specimens for HRTEM observation were prepared by a dual-beam scanning electron microscopy (SEM)/focused ion beam (FIB) system (FEI Quanta 3D FEG and FEI Helios) based on site-specific in situ lift-out technique. Before the ion thinning, a metallic protective layer of $\mathrm{Cr}$ with a thickness between 30 and $40 \mathrm{~nm}$ was first deposited on the sample surface. Then, a platinum supporting layer with a thickness between 1 and $1.5 \mu \mathrm{m}$ was further deposited on the targeted area using the ion beam deposition inside the chamber This dual-beam system is capable of fast milling at a high voltage of $30 \mathrm{kV}$ and subtle thinning at the final stage using a few $\mathrm{kV}(2-5 \mathrm{kV}) \mathrm{Ga}^{+}$ion milling. During ion thinning, the sample was tilted between 1 and $2^{\circ}$ towards the ion beam in order to avoid significant damage to the sample structure. The ion beam current was between 10 and $15 \mathrm{pA}$.

X-ray diffraction Characterization. X-ray diffraction was done with a Panalytical X'Pert PRO MPD system with a $\mathrm{Cu} \mathrm{Ka}$ source and a hybrid mirror with a twobounce two-crystal $\mathrm{Ge}(220)$ monochromator, yielding a parallel incident beam with a wavelength $\lambda_{\mathrm{Ka} 1}=1.5406 \AA$, a divergence of $0.0068^{\circ}$, and a width of $0.3 \mathrm{~mm}$. Sample alignment included height adjustment as well as correction of the $\omega$ and $\chi$ tilt angles by maximizing the substrate peak intensity. Short-range symmetric $\omega$ $-2 \theta$ scans were obtained using a 0.04 radian Soller slit in front of a PIXcel solidstate line detector operated in receiving mode with a $0.165 \mathrm{~mm}$ active length, corresponding to a $2 \theta$ opening of less than $0.04^{\circ}$. $\omega$-rocking curves were obtained using constant $2 \theta$ angles corresponding to $\mathrm{CsPbBr}_{3} 004$ reflections (indexed with tetragonal structure $P 4 / \mathrm{mbm}$ ) and using the same parallel beam geometry as used for $\omega-2 \theta$ scans. Asymmetric high-resolution reciprocal space maps (HR RSM) around 224 reflections (indexed with pseudocubic to simplify the analysis) were obtained using a line detector in scanning mode, operating all 255 channels. This is done using a small angle $\left(\sim 20^{\circ}\right)$ between the sample surface and the diffracted beam to cause a beam narrowing which increases the $2 \theta$ resolution and therefore facilitates fast high-resolution reciprocal space mapping. Then the data was shown as color filled iso-intensity contour maps in a logarithmic scale, plotted within $k$ space where $k_{\perp}=2 \sin \theta \cos (\omega-\theta) / \lambda$ and $k_{\|}=2 \sin \theta \sin (\omega-\theta) / \lambda$ correspond to directions perpendicular and parallel to the substrate surface along perpendicular [001] and [110] directions, respectively. XRD pole figures were obtained using a point focus optics with a poly-capillary $\mathrm{x}$-ray lens that provides a quasi-parallel $\mathrm{Cu}$ $\mathrm{Ka}$ beam with a divergence of less than $0.3^{\circ}$ to minimize defocusing effects associated with the non-uniform sample height due to the sample tilt. In addition, $\omega-2 \theta$ scans with a divergent beam Bragg-Brentano geometry were acquired over a large $2 \theta$ range from $5-90^{\circ}$ in order to detect small inclusions of possible secondary phases or misoriented grains. 
Microscopy characterization. Morphology of the halide perovskite thin films and flakes was characterized by a Nikon Eclipse Ti-S inverted optical microscope. Scanning Electron Microscope (SEM) FEI Versa 3D was used for surface morphology and thickness (cross-sectional SEM) analysis. The high resolution TEM images and electron diffraction pattern for transferred $\mathrm{CsPbBr}_{3}$ flakes were collected with FEI F20 TEM operated at $200 \mathrm{kV}$. The cross-sectional interfacial morphologies and crystallite structures were evaluated by high resolution transmission electron microscope (HRTEM) JEM2010 operating at $120 \mathrm{kV}$, JEOL 2100 $\mathrm{F}$ operating at $200 \mathrm{kV}$ and scanning transmission electron microscope (STEM) FEI Titan G2 80-200 CREWLEY operating at $80 \mathrm{kV}$.

High resolution atomic force microscopy (HRAFM). The surface morphologies were investigated by high resolution atomic force microscopy (AFM, Cypher ES, Oxford Instruments) in ambient air. To derive the atomic-scale structures of the crystallite surface, a sharp $\mathrm{Si}_{3} \mathrm{~N}_{4}$ probe (TR400PB, Olympus) was used in the contact mode. The lateral force signal was recorded in order to atomically resolve the recorded image as the friction force is sensitive to the evolution of surface crystal lattice. To avoid surface damage, the applied normal load was controlled in the range of $10-20 \mathrm{nN}$ and the sliding velocity was set at $10-15 \mathrm{~Hz}$. The recorded image was processed by the Asylum Research Igor Pro Software.

Piezoelectric force microscopy (PFM). The piezoelectrics properties of the samples were measured by piezoelectric force microscopy (PFM) in an AFM system (MFP 3D, Oxford Instruments). A conductive probe with platinum overall coating (ElectriMulti75-G, BudgetSensors) was used, with a drive voltage up to $10 \mathrm{~V}$ applied to the conductive tip. When the tip was brought in contact with the sample surface, the local piezoelectric response can be detected by recording the distortional motion of the cantilever. Thus the piezoresponse can be estimated measuring the vibrating amplitude of the cantilever per unit drive voltage. The scanning velocity was $1 \mathrm{~Hz}$. Both the amplitude and phase signals of the piezoresponse within a specific domain were acquired.

Raman spectroscopy. Confocal Raman spectroscopy was performed using a Horiba XploRA Nano system. One of two excitation wavelengths, $532 \mathrm{~nm}$ and $638 \mathrm{~nm}$, was utilized based on the needs of different situations. A typical Raman spectrum was collected under following conditions: $100 \mathrm{X}$ objective lens, $3 \mathrm{~s}$ integration time, 10 acquisitions, 1200 grooves $/ \mathrm{mm}$ grating, $4 \mathrm{~mW}$ laser power.

Photoluminescence (PL) characterization. The PL of samples were measured via a customized PL system consisting of a Picoquant $405 \mathrm{~nm}$ pulsed laser with a $2 \mathrm{~mW}$ power and a repetition of $4 \mathrm{MHz}$, a Thorlabs 4 Megapixel Monochrome Scientific CCD Camera, a Princeton Instruments SP-2358 spectrograph, and the same optical microscope that focuses the laser via a $\times 50$ objective lens.

Time resolved photoluminescence (TRPL) characterization. Time resolved PL was measured in the same PL system. A Picoquant PDM series single-photon detector synchronized with the pulsed laser source was used to collect the time domain PL information.

DFT calculation. The DFT calculations were performed with the Vienna ab initio Simulation Package (VASP) ${ }^{67}$. The core electrons were described by the projectoraugmented-wave (PAW) method ${ }^{68}$ and the electron exchange and correlation were modeled within the generalized gradient approximation (GGA) using the PerdewBurke-Ernzerhof (PBE) form ${ }^{69}$. The non-local optB86b-vdW exchange-correlation functional was used to describe the dispersion interaction (vdW forces) approximately ${ }^{70}$, as its accurate descriptions of geometrical structures and energies. The plane wave basis kinetic energy cut off was set to $400 \mathrm{eV}$.

For $\mathrm{CsPbBr}_{3}(001)$ growth on $\mathrm{Gr} / \mathrm{NaCl}(001)$, the calculation supercells were constructed as four layers of $\mathrm{CsPbBr}_{3}(001)(a=5.89 \AA$, cubic phase is chosen for calculation) sitting on four layers of $\mathrm{NaCl}(001)(a=5.57 \AA)$, with about $5 \%$ compressive strain imposing on $\mathrm{CsPbBr}_{3}(001)$. The remote atomic interaction of $\mathrm{NaCl}(001)$ though monolayer graphene was simulated with $4 \times 3 \mathrm{CsPbBr}_{3}$ sitting on monolayer graphene-coated $4 \times 3 \mathrm{NaCl}(001)$. The selection of this model is mainly considering to decrease strain on graphene intercalation. The top $\mathrm{NaCl}$ (001) layer and atoms above were allowed to relax until the forces on all the relaxed atoms were less than $0.05 \mathrm{eV} / \AA$. The vacuum gaps in simulation supercells are larger than $10 \AA$.

For $\mathrm{CsPbBr}_{3}(011)$ growth on $\mathrm{Gr} / \mathrm{CaF}_{2}(001)$, the calculation supercell was constructed as three layers of $\mathrm{CsPbBr}_{3}(011)(a=5.89 \AA, b=8.33 \AA)$ sitting on three layers of $\mathrm{CaF}_{2}(001)(a=5.35 \AA)$. The remote atomic interaction of $\mathrm{CaF}_{2}(001)$ through monolayer graphene was simulated with $7 \mathrm{a} \times 2 \mathrm{~b} \mathrm{CsPbBr}_{3}(011)$ sitting on monolayer graphene-coated $8 \times 3 \mathrm{CaF}_{2}(001)$. The selection of this model is mainly based on the minimization of the mismatch between $\mathrm{CsPbBr}_{3}$ and $\mathrm{CaF}_{2}(001)$. For the epitaxial relation of $\mathrm{CsPbBr}_{3}(011) \| \mathrm{CaF}_{2}(001)$, the mismatch is $3.8 \%$ along $\mathrm{CsPbBr}_{3}[100] \| \mathrm{CaF}_{2}[100]$ and $-3.7 \%$ along $\mathrm{CsPbBr}_{3}[0 \overline{1} 1] \| \mathrm{CaF}_{2}[010]$, which is remarkably smaller than the mismatch of $-9 \%$ along $\mathrm{CsPbBr}_{3}[100] \| \mathrm{CaF}_{2}[100]$ for $\mathrm{CsPbBr}_{3}(001) \| \mathrm{CaF}_{2}(001)$. The strain imposed on graphene is less than $2 \%$. The top $\mathrm{CaF}_{2}(001)$ layer and atoms above were allowed to relax until the forces on all the relaxed atoms were less than $0.05 \mathrm{eV} / \AA ̊$. The vacuum gap in simulation supercell is larger than $20 \AA$

Molecular dynamic simulation. We designed a minimalist's molecular-level model to simulate the deposition and subsequent epitaxial growth of a model perovskite film on various substrates. This model enables us to investigate structural features of a three-component epitaxial film such as misfit dislocations with moderate computational resources. In addition, observations made with such a generic perovskite model are likely to be valid for perovskite systems in general, as opposed to observations stemming from unique features of a particular material.

There are three particle species (A, B, and C) in the model perovskite crystal $\mathrm{ABC}_{3}$. The particle-particle interaction is described by a truncated Lennard-Jones (LJ) potential:

$$
\phi_{\mathrm{LJ}}(r)=4 \varepsilon_{\alpha \beta}\left(\frac{\sigma_{\alpha \beta}^{12}}{r^{12}}-\frac{\sigma_{\alpha \beta}^{6}}{r^{6}}\right)-4 \varepsilon_{\alpha \beta}\left(\frac{\sigma_{\alpha \beta}^{12}}{r_{\alpha \beta, \mathrm{c}}^{12}}-\frac{\sigma_{\alpha \beta}^{6}}{r_{\alpha \beta, \mathrm{c}}^{6}}\right)
$$

We map the generic $\mathrm{ABC}_{3}$ perovskite to $\mathrm{CsPbBr}$ to obtain the mass and size of each particle. It should be noted that we do not intend to use LJ potential to describe the complex interactions including long-range Coulombic force within $\mathrm{CsPbBr}$. Yet, even $\mathrm{LJ}$ potential, with the proper choice of bond strengths together with the bond length in $\mathrm{CsPbr}_{3}$, leads to the crystallization of perovskite. The pair-dependent bond strength, bond length, and cutoff are listed in Supplementary Table 1. The energy and length units are chosen as $\varepsilon_{\mathrm{BC}}$ and $\sigma_{\mathrm{BC}}$ for convenience. Given a unit mass of $\mathrm{m}_{0}$, the particle mass for $\mathrm{A}, \mathrm{B}$ and $\mathrm{C}$ are $1.66,2.6$ and $1.0 \mathrm{~m}_{0}$, respectively.

Consequently, the temperature unit is $\frac{\varepsilon_{\mathrm{BC}}}{k_{\mathrm{B}}}\left(k_{\mathrm{B}}\right.$ is the Boltzmann constant), and the time unit is $\tau_{0}=\sigma_{\mathrm{BC}} \sqrt{\frac{m_{0}}{\varepsilon_{\mathrm{BC}}}}$. The melting point of the $\mathrm{ABC}_{3}$ perovskite was estimated to be about $0.13 \frac{\varepsilon_{\mathrm{B}}}{k_{\mathrm{B}}}$. Therefore, the reduced units above for this model perovskite system can be written in real units by mapping to $\mathrm{CsPbBr}{ }_{3}$ as, $\varepsilon_{\mathrm{BC}}=$ $0.557 \mathrm{eV}$ (using the melting point of $\mathrm{CsPbBr}$ as $567^{\circ} \mathrm{C}$ ), $\sigma_{\mathrm{BC}}=0.249 \mathrm{~nm}$ (using the lattice constant of $\mathrm{CsPBr}_{3}$ as $0.587 \mathrm{~nm}$ ), $\mathrm{m}_{0}=79.9 \mathrm{amu}$ (using the mass of $\mathrm{Br}$ ), and $\tau_{0}=0.3 \mathrm{ps}$.

The perovskite potential parameters and the rational are discussed as follows. Only B-C and A-C interactions have attractive regime, while all other pair interactions are repulsive-only (the cutoffs are set at the bottom of the LJ potentia well). $\mathrm{B}-\mathrm{C}$ pairs have strong affinity, together with the $\mathrm{C}-\mathrm{C}$ repulsion (and the proper hardsphere size), lead to BC6 octohedral formation. A-C pairs have weaker attraction than $\mathrm{B}-\mathrm{C}$ pairs to favor $\mathrm{ABC}_{3}$ perovskite formation rather than $\mathrm{B}-\mathrm{C}$ compound formation. The lattice constant of the ideal perovskite structure is about $2.2449 \sigma_{\mathrm{BC}}$ at zero temperature, and around $2.36 \sigma_{\mathrm{BC}}$ at the deposition temperature of $0.1 \varepsilon_{\mathrm{BC}} / k_{\mathrm{B}}$. The temperature is controlled by Nose-Hoover thermostat. The equations of motion are numerically integrated using the velocity-Verlet algorithm with a time step of $0.0056 \tau_{0}$.

We have investigated three different types of substrates for the deposition of perovskite $\mathrm{ABC}_{3}$. The first type of substrate (termed Substrate I) has a strong affinity with the deposited film, which is analogous to 'ionic epitaxy'. There are two types of particles (D and E particles) forming a rigid substrate. E particles form a square lattice with a lattice constant of $1.1352 \sigma_{\mathrm{BC}}$. One quarter of the $\mathrm{E}$ particles were replaced by $\mathrm{D}$ particles such that $\mathrm{D}$ particles themselves form a square lattice with a lattice constant of $2.2704 \sigma_{\mathrm{BC}}$. Due to the strong affinity between C and D particles, epitaxy growth is expected with a lattice mismatch of about $3.8 \%$ at the deposition temperature. The second type of substrate (termed Substrate II) is identical to Substrate I with an additional model 2D material (graphene-like) layer acting as a spacer so as to weaken the substrate-film affinity, which is analogous to 'remote epitaxy'. The model $2 \mathrm{D}$ material has a honeycomb lattice made of $\mathrm{F}$ particles, with atomic spacing of $0.971 \sigma_{\mathrm{BC}}$, which is $0.925 \sigma_{\mathrm{BC}}$ away from the original substrate. There is a very weak attraction between $\mathrm{C}$ and $\mathrm{F}$ particles to facilitate deposition. The third type of substrate (termed Substrate III) has a weak affinity to the film (identical to Substrate I except the C-D interaction is only half as strong), which is analogous to 'van der Waals epitaxy'.

The simulation system for deposition is around $113.5 \sigma_{B C}$ by $113.5 \sigma_{\mathrm{BC}}$ by 90.8 $\sigma_{\mathrm{BC}}$ for simulations with Substrate I and III, and $106.7 \sigma_{\mathrm{BC}}$ by $113.5 \sigma_{\mathrm{BC}}$ by 90.8 $\sigma_{\mathrm{BC}}$ for simulations with Substrate II. During deposition, all atoms belong to the substrate are frozen for simplicity. For all deposition simulations, particles A, B and $\mathrm{C}$ are created randomly without overlapping with any existing atoms at the top of the simulation box. On average, particles A and B are created every 600 timesteps (or $3.36 \tau_{0}$ ), while particles $\mathrm{C}$ are created every $1.12 \tau_{0}$ to maintain the correct stoichiometry. All particles are given an initial velocity towards the substrate. The deposition temperature is set to be $0.1 \varepsilon_{\mathrm{BC}} / k_{\mathrm{B}}$ (about $77 \%$ of the melting point).

\section{Data availability}

All the relevant data are available from the corresponding authors upon reasonable request.

\section{Code availability}

All the relevant code are available from the corresponding authors upon reasonable request. 
Received: 1 February 2019 Accepted: 20 August 2019.

Published online: 12 September 2019

\section{References}

1. Schubert, M. F. et al. Effect of dislocation density on efficiency droop in GaInN/GaN light-emitting diodes. Appl. Phys. Lett. 91, 231114 (2007).

2. Nakamura, S. The roles of structural imperfections in InGaN-based blue lightemitting diodes and laser diodes. Science 281, 956-961 (1998).

3. Yamaguchi, M., Yamamoto, A. \& Itoh, Y. Effect of dislocations on the efficiency of thin-film GaAs solar cells on Si substrates. J. Appl. Phys. 59, 1751-1753 (1986).

4. Kumakura, K. et al. Minority carrier diffusion length in GaN: dislocation density and doping concentration dependence. Appl. Phys. Lett. 86, 052105 (2005).

5. Kieliba, T., Riepe, S. \& Warta, W. Effect of dislocations on minority carrier diffusion length in practical silicon solar cells. J. Appl. Phys. 100, 063706 (2006).

6. Chen, Z., Guo, Y., Wertz, E. \& Shi, J. Merits and challenges of ruddlesden-popper soft halide perovskites in electro-optics and optoelectronics. Adv. Mater. 31, 1803514 (2019).

7. Manser, J. S., Christians, J. A. \& Kamat, P. V. Intriguing optoelectronic properties of metal halide perovskites. Chem. Rev. 116, 12956 (2016).

8. Yang, W. S. et al. Iodide management in formamidinium-lead-halide-based perovskite layers for efficient solar cells. Science 356, 1376-1379 (2017).

9. Xiao, Z. et al. Giant switchable photovoltaic effect in organometal trihalide perovskite devices. Nat. Mater. 14, 193-198 (2015)

10. McMeekin, D. P. et al. A mixed-cation lead mixed-halide perovskite absorber for tandem solar cells. Science 351, 151-155 (2016).

11. Yuan, M. et al. Perovskite energy funnels for efficient light-emitting diodes. Nat. Nanotechnol. 11, 872 (2016).

12. Wang, N. et al. Perovskite light-emitting diodes based on solution-processed self-organized multiple quantum wells. Nat. Photonics 10, 699 (2016).

13. $\mathrm{Zhu}, \mathrm{H}$. et al. Lead halide perovskite nanowire lasers with low lasing thresholds and high quality factors. Nat. Mater. 14, 636 (2015).

14. Ha, S.-T., Shen, C., Zhang, J. \& Xiong, Q. Laser cooling of organic-inorganic lead halide perovskites. Nat. Photonics 10, 115 (2015).

15. Xing, G. et al. Low-temperature solution-processed wavelength-tunable perovskites for lasing. Nat. Mater. 13, 476-480 (2014).

16. Fang, Y., Dong, Q., Shao, Y., Yuan, Y. \& Huang, J. Highly narrowband perovskite single-crystal photodetectors enabled by surface-charge recombination. Nat. Photonics 9, 679 (2015).

17. Dou, L. et al. Solution-processed hybrid perovskite photodetectors with high detectivity. Nat. Commun. 5, 5404 (2014).

18. Senanayak S. P. et al. Understanding charge transport in lead iodide perovskite thin-film field-effect transistors. Sci. Adv. 3, e1601935 (2017)

19. Li, F. et al. Ambipolar solution-processed hybrid perovskite phototransistors. Nat. Commun. 6, 8238 (2015).

20. Walsh, A. \& Zunger, A. Instilling defect tolerance in new compounds. Nat. Mater. 16, 964 (2017).

21. Meggiolaro, D. et al. Iodine chemistry determines the defect tolerance of leadhalide perovskites. Energy Environ. Sci. 11, 702-713 (2018).

22. Yin, W.-J., Shi, T. \& Yan, Y. Unusual defect physics in CH3NH3PbI3 perovskite solar cell absorber. Appl. Phys. Lett. 104, 063903 (2014).

23. Park, N.-G., Grätzel, M., Miyasaka, T., Zhu, K. \& Emery, K. Towards stable and commercially available perovskite solar cells. Nat. Energy 1, 16152 (2016).

24. Son, D.-Y. et al. Self-formed grain boundary healing layer for highly efficient CH3 NH3 PbI3 perovskite solar cells. Nat. Energy 1, 16081 (2016).

25. Long, R., Liu, J. \& Prezhdo, O. V. Unravelling the effects of grain boundary and chemical doping on electron-hole recombination in $\mathrm{CH}_{3} \mathrm{NH}_{3} \mathrm{PbI}_{3}$ perovskite by time-domain atomistic simulation. J. Am. Chem. Soc. 138, 3884-3890 (2016).

26. Shao, Y. et al. Grain boundary dominated ion migration in polycrystalline organic-inorganic halide perovskite films. Energy Environ. Sci. 9, 1752-1759 (2016).

27. de Quilettes, D. W. et al. Impact of microstructure on local carrier lifetime in perovskite solar cells. Science 348, 683-686 (2015).

28. Yang, Y. et al. Low surface recombination velocity in solution-grown $\mathrm{CH}_{3} \mathrm{NH}_{3} \mathrm{PbBr}_{3}$ perovskite single crystal. Nat. Commun. 6, 7961 (2015).

29. Yang, Y. et al. Top and bottom surfaces limit carrier lifetime in lead iodide perovskite films. Nat. Energy 2, 16207 (2017)

30. Leijtens, T. et al. Carrier trapping and recombination: the role of defect physics in enhancing the open circuit voltage of metal halide perovskite solar cells. Energy Environ. Sci. 9, 3472-3481 (2016).

31. Ball, J. M. \& Petrozza, A. Defects in perovskite-halides and their effects in solar cells. Nat. Energy 1, 16149 (2016)
32. Azpiroz, J. M., Mosconi, E., Bisquert, J. \& De Angelis, F. Defect migration in methylammonium lead iodide and its role in perovskite solar cell operation. Energy Environ. Sci. 8, 2118-2127 (2015).

33. Rehman, W. et al. Charge-carrier dynamics and mobilities in formamidinium lead mixed-halide perovskites. Adv. Mater. 27, 7938-7944 (2015).

34. He, H. et al. Exciton localization in solution-processed organolead trihalide perovskites. Nat. Commun. 7, 10896 (2016)

35. Juarez-Perez, E. J. et al. Photoinduced giant dielectric constant in lead halide perovskite solar cells. J. Phys. Chem. Lett. 5, 2390-2394 (2014).

36. Zhu, H. et al. Screening in crystalline liquids protects energetic carriers in hybrid perovskites. Science 353, 1409-1413 (2016).

37. Shi, D. et al. Low trap-state density and long carrier diffusion in organolead trihalide perovskite single crystals. Science 347, 519-522 (2015).

38. Dong, Q. et al. Electron-hole diffusion lengths $>175 \mu \mathrm{m}$ in solution grown $\mathrm{CH}_{3} \mathrm{NH}_{3} \mathrm{PbI}_{3}$ single crystals. Science 347, 967-970 (2015).

39. Herz, L. M. Charge-carrier dynamics in organic-inorganic metal halide perovskites. Annu. Rev. Phys. Chem. 67, 65-89 (2016).

40. Kim, Y. et al. Remote epitaxy through graphene enables two-dimensional material-based layer transfer. Nature 544, 340 (2017).

41. Kong, W. et al. Polarity governs atomic interaction through two-dimensional materials. Nat. Mater. 17, 999-1004 (2018).

42. Qi, Y. et al. Fast Growth of Strain-Free AlN on Graphene-Buffered Sapphire. J. Am. Chem. Soc. 140, 11935-11941 (2018).

43. $\mathrm{Lu}, \mathrm{Z}$. et al. Remote epitaxy of copper on sapphire through monolayer graphene buffer. Nanotechnology 29, 445702 (2018)

44. Jeong, J. et al. Remote heteroepitaxy across graphene: hydrothermal growth of vertical $\mathrm{ZnO}$ microrods on graphene-coated GaN substrate. Appl. Phys. Lett. 113, 233103 (2018).

45. Kim, J. et al. Principle of direct van der Waals epitaxy of single-crystalline films on epitaxial graphene. Nat. Commun. 5, 4836 (2014).

46. Chen, Z. et al. High-brightness blue light-emitting diodes enabled by a directly grown graphene buffer layer. Adv. Mater. 30, 1801608 (2018).

47. Malard, L. M., Pimenta, M. A., Dresselhaus, G. \& Dresselhaus, M. S. Raman spectroscopy in graphene. Phys. Rep. 473, 51-87 (2009)

48. Stoumpos, C. C. et al. Crystal growth of the perovskite semiconductor $\mathrm{CsPbBr3}$ : a new material for high-energy radiation detection. Cryst. Growth Des. 13, 2722-2727 (2013).

49. Rodová, M., Brožek, J., Knížek, K. \& Nitsch, K. Phase transitions in ternary caesium lead bromide. J. Therm. Anal. Calorim. 71, 667-673 (2003).

50. Wang, Y., Shi, Y., Xin, G., Lian, J. \& Shi, J. Two-dimensional van der Waals Epitaxy kinetics in a three-dimensional perovskite halide. Cryst. Growth Des. 15, 4741-4749 (2015)

51. Yaffe, O. et al. Local polar fluctuations in lead halide perovskite crystals. Phys Rev. Lett. 118, 136001 (2017).

52. Zhou, Y., Sternlicht, H. \& Padture, N. P. Transmission electron microscopy of halide perovskite materials and devices. Joule 3, 641-661 (2019).

53. Luo Y.-R. Comprehensive handbook of chemical bond energies (CRC press, 2007).

54. Wang, Y. et al. High-temperature ionic epitaxy of halide perovskite thin film and the hidden carrier dynamics. Adv. Mater. 29, 1702643 (2017).

55. Distler, G. I. \& Zvyagin, B. B. A dislocation-free mechanism of growth of real crystals. Nature 212, 807-808 (1966).

56. Matthews, J. W. \& Blakeslee, A. E. Defects in epitaxial multilayers: I. Misfit dislocations. J. Cryst. Growth 27, 118-125 (1974).

57. Chen, H. et al. Low-temperature buffer layer for growth of a low-dislocationdensity SiGe layer on Si by molecular-beam epitaxy. J. Appl. Phys. 79, 1167-1169 (1996)

58. Chen, Z. et al. Regulating carrier dynamics in single crystal halide perovskite via interface engineering and optical doping. Adv. Electron. Mater. 2, 1600248 (2016).

59. Wehrenfennig, C., Eperon, G. E., Johnston, M. B., Snaith, H. J. \& Herz, L. M. High charge carrier mobilities and lifetimes in organolead trihalide perovskites. Adv. Mater. 26, 1584-1589 (2014).

60. Harding, W. R., Blenkinsop, I. D. \& Wight, D. R. Dislocation-limited minority-carrier lifetime in n-type GaP. Electron. Lett. 12, 503-504 (1976)

61. Wight, D. R., Blenkinsop, I. D., Harding, W. \& Hamilton, B. Diffusion-limited lifetime in semiconductors. Phys. Rev. B 23, 5495-5510 (1981).

62. Sproul, A. B. Dimensionless solution of the equation describing the effect of surface recombination on carrier decay in semiconductors. J. Appl. Phys. 76, 2851-2854 (1994)

63. Chen, J. et al. Single-crystal thin films of cesium lead bromide perovskite epitaxially grown on metal oxide perovskite (SrTiO3). J. Am. Chem. Soc. 139 13525-13532 (2017).

64. Mendelson, S. Dislocation etch pit formation in sodium chloride. J. Appl. Phys. 32, 1579-1583 (1961).

65. Pernot, C. et al. Low-Intensity ultraviolet photodetectors based on AlGaN. Jpn. J. Appl. Phys. 38, L487-L489 (1999). 
66. Sun, X. et al. van der Waals epitaxy of antimony islands, sheets, and thin films on single-crystalline graphene. ACS Nano 12, 6100-6108 (2018).

67. Kresse, G. \& Furthmüller, J. Efficient iterative schemes for ab initio total-energy calculations using a plane-wave basis set. Phys. Rev. B 54, 11169-11186 (1996).

68. Blöchl, P. E. Projector augmented-wave method. Phys. Rev. B 50, 17953-17979 (1994).

69. Perdew, J. P., Burke, K. \& Ernzerhof, M. Generalized gradient approximation made simple. Phys. Rev. Lett. 77, 3865-3868 (1996).

70. Klimeš, J., Bowler, D. R. \& Michaelides, A. Van der Waals density functionals applied to solids. Phys. Rev. B 83, 195131 (2011).

\section{Acknowledgements}

We acknowledge the financial support from the National Key Research and Development Program of China (Grant No. 2017YFB0702100), National Natural Science Foundation of China (Grant No. 51705017, U1706221, and 51727901). The experimental work is supported by the NYSTAR Focus Center at Rensselaer Polytechnic Institute (RPI), C150117, NSF award under No. 1635520, No. 1712752, No. 1508410 and No. 1706815, Air Force Office of Scientific Research under award number FA9550-18-1-0116, the Office of Naval Research under award number N000141812408 and NSF MIP PARADIM under award number 1539918. Research performed at Brown University was supported by the National Science Foundation (OIA-1538893) and the Office for Naval Research (N00014-17-1-2232). K.B. and H.T. are grateful to the National Science Foundation (EFRI-1433311), the Center for Computational Innovations (CCI) at Rensselaer Polytechnic Institute, and the Extreme Science and Engineering Discovery Environment (XSEDE, project TG-DMR17008), which is supported by National Science Foundation grant number ACI-1053575.

\section{Author contributions}

J.S. conceived the idea. J.S., J.F. and L.G. supervised the project. J.J., X.S. and J.S. designed the experiments. X.S. processed and prepared substrates coated with graphene. J.J. prepared flakes/thin films and performed SEM. J.J. and Z.C. performed PL measurements. J.J., Z.C. and Y.H. performed device fabrication and photo response measurements. X.S., K.B. and H.T. performed Raman spectroscopy measurements and analysis. B.W. performed XRD. J.J. and Y.H. analyzed the domain pattern. L.Z. performed AFM. Y.G. performed HRTEM at Cornell University. F.Z. and L.J. performed STEM. J.J., J.S., M.C.,
Z.M., Y.Z. and N.P.P. performed FIB and TEM at Brown University. X.C. performed FIB, HRAFM and PFM at Tsinghua University. Y.M. and L.G. performed DFT calculations. Y.S. performed MD simulations. J.J. analyzed data and wrote the paper. All the authors were involved in the discussion for data analysis. D.G., X.S., T.L., E.W. and J.S. revised the manuscript.

\section{Additional information}

Supplementary Information accompanies this paper at https://doi.org/10.1038/s41467019-12056-1.

Competing interests: The authors declare no competing interests.

Reprints and permission information is available online at http://npg.nature.com/ reprintsandpermissions/

Peer review information Nature Communications thanks the anonymous reviewers for their contribution to the peer review of this work. Peer reviewer reports are available.

Publisher's note Springer Nature remains neutral with regard to jurisdictional claims in published maps and institutional affiliations.

Open Access This article is licensed under a Creative Commons Attribution 4.0 International License, which permits use, sharing, adaptation, distribution and reproduction in any medium or format, as long as you give appropriate credit to the original author(s) and the source, provide a link to the Creative Commons license, and indicate if changes were made. The images or other third party material in this article are included in the article's Creative Commons license, unless indicated otherwise in a credit line to the material. If material is not included in the article's Creative Commons license and your intended use is not permitted by statutory regulation or exceeds the permitted use, you will need to obtain permission directly from the copyright holder. To view a copy of this license, visit http://creativecommons.org/ licenses/by/4.0/

(C) The Author(s) 2019 